\title{
OBESITY: DEVELOPMENT, EPIDEMIOLOGY, FACTORS AFFECTING, QUANTITY, HEALTH HAZARDS, MANAGEMENT AND NATURAL TREATMENT-A REVIEW
}

\author{
MANI KUMAR BOLLAPRAGADA ${ }^{1}$, MANJULA SHANTARAM ${ }^{1}$, SUNIL KUMAR R. ${ }^{2}$
}

${ }^{1}$ Department of Studies in Biochemistry, Mangalore University, Post Graduate Centre, Chikka Aluvara, Kodagu, Karnataka, 571232 India, ${ }^{2}$ Department of Biotechnology, College of Agriculture, University of Agriculture Science, Bangalore, Hassan, Karnataka, 573225 India Email: manjula59@gmail.com

Received: 04 Sep 2016 Revised and Accepted: 05 Jan 2017

\begin{abstract}
Due to the advancement in science, enhanced knowledge on the physiological aspects of almost all the tissues and the organs of the human body is gained. One of the most important prevalent topics needed for discussion is obesity and its effect on the metabolic changes leading to disorders in the human body such as diabetes, hypertension, cardiovascular diseases in addition to chronic diseases such as stroke, osteoarthritis, sleep apnea, some cancers, and inflammation-based pathologies. In recent years, obesity is a serious socioeconomic issue, which has become one of the major health problems all over the world, affecting people of all ages, sex, ethnicities and races. Obesity is a complex and multifactorial disease caused by the interaction of a myriad genetics, dietary, lifestyle and environmental factors and it is characterised by an excessive weight for height due to an enlarged fat deposition in the adipose tissue, which is due to a higher calorie intake than the energy expenditure. The pharmaceutical drugs are currently available to treat obesity but generally they have unpleasant side effects. Recent researches demonstrated the potential of natural products to counteract on obesity. Now the novel promising approach is the usage of dietary supplements and plant products and their bioactive compounds that could interfere on pancreatic lipase activity, food intake, lipid metabolism and adipocyte differentiation. In a similar way, hundreds of extracts are currently being isolated from plants, fungi, algae or bacteria and are screened for their potential inhibitions of activity against obesity. Natural products may have a synergistic activity that increases their bioavailability and action on multiple molecular targets.
\end{abstract}

Keywords: Obesity, Anti-obesity, Lipase inhibitors, Pancreatic lipase, Adipocyte, Appetite suppressant

(c) 2016 The Authors. Published by Innovare Academic Sciences Pvt Ltd. This is an open access article under the CC BY license (http://creativecommons.org/licenses/by/4. 0/) DOI: http://dx.doi.org/10.22159/ijpps.2017v9i2.15049

\section{INTRODUCTION}

Obesity has become a major health problem worldwide, affecting people across all ages, sex, ethnicities, and races. Obesity incidence is increased at an alarming rate and is becoming a major public health concern. Indeed, obesity facilitates the development of metabolic disorders and cardiovascular diseases in addition to chronic diseases $[1,2]$. Body mass index (BMI), defined as the weight in kilogrammes divided by the height in meters squared $\left(\mathrm{kg} / \mathrm{m}^{2}\right)$, is the most widely used measure of obesity due to its low cost and simplicity. The World Health Organization (WHO) and the National Institutes of health $(\mathrm{NIH})$, have defined overweight as having a BMI between 25.0 and $29.9 \mathrm{~kg} / \mathrm{m}^{2}$ and obesity as having a BMI greater than $30.0 \mathrm{~kg} / \mathrm{m}^{2}$ [3]. Some recent WHO global estimates in 2014, more than 1.9 billion adults aged $18 \mathrm{y}$ and older were overweight. Of these, over 600 million adults were obese. In 2014, an estimated 41 million children under the age of $5 \mathrm{y}$ were overweight or obese. Once considered a high-income country problem, overweight and obesity are now on the rise in low and middle-income countries, particularly in urban settings. In Africa, the number of children who are overweight or obese has nearly doubled from 5.4 million in 1990 to 10.6 million in 2014. Nearly half of the children under $5 \mathrm{y}$ who were overweight or obese in 2014 lived in Asia. Obesity is linked to more deaths worldwide than underweight. Globally there are more people who are obese than underweight. This occurs in every region except parts of sub-Saharan Africa and Asia [4].

According to research in different countries an obese individual incurs health care expenditures at least $25 \%$ higher than a healthy person [5]. The progress of obesity is characterised by a chronic imbalance of energy intake and energy expenditure [6, 7]. In addition, decreased energy expenditure is often associated with an inherited low basal metabolic rate and low energy cost of physical activity and low capacity for fat oxidation [8]. To reduce body weight and adiposity, a change in lifestyle habits is still the crucial keystone [9]. Physical activity might be the key step in the prevention of obesity by increasing average daily metabolic rate and increased energy expenditure [6]. Unfortunately, this clinical approach is not long lasting and weight regain often seen. Drugs that prevent weight regain appear necessary in obesity treatment [9]. Thus, the development of natural products to treat obesity is a challenging task, which can be launched faster and cheaper than conventional single-entity pharmaceuticals [10]. Many medicinal plants may offer safe, natural, and cost-effective alternatives to synthetic drugs [1117]. Currently, one of the most significant strategies in the treatment of obesity comprises the development of natural inhibitors for nutrient digestion, absorption and its sequential metabolic reaction pathways [18]. For example, some recent studies are: Ginsenoside F2 and Rh1 from Panax ginseng Meyer plant possesses anti-obesity activity via binding with peroxisome proliferator activated receptor$\gamma$ (PPAR $\gamma$ ) and inhibiting adipocyte differentiation in the 3T3-L1 cell line $[19,20]$. Raspberry ketone, a naturally occurring phenolic compound inhibits adipogenic and lipogenic gene expression in 3T3L1 adipocytes [21]. Aqueous tuber extract of Cyperus rotundus L., corn silk extracts show a significant decrease in obesity [22, 23].

In this context, dietary lipids represent the major source of unwanted calories, the attenuation of this fat digestion is an interesting approach for reducing fat absorption [24, 25]. Orlistat is the only authorised anti-obesity drug approved from food and drug administration (FDA). It has been shown to act through inhibition of pancreatic lipase (PL), which is a key enzyme for the digestion of dietary triglycerides [26]. Orlistat is a saturated derivative of lipastatin, an inhibitor of PL isolated from the bacterium Streptomyces toxytricini [27]. This molecule exerts a modest weight lowering effect while accompanying a suitable dietary advice. Thus, in a recent meta-analysis [28], the mean BMI change with Orlistat (120 mg three times daily) was a reduction of $0.83 \mathrm{~kg} / \mathrm{m}^{2}$ compared with a placebo. Accompanying this anti-obesity action, Orlistat is also able to modestly reduce blood pressure, improve oral glucose tolerance and prevent the onset of type 2 diabetes $[29,30]$. Now, extracts from hundreds of species of medicinal plants, vegetables and fruits [31] as well as products from microorganisms [11], fungi [32], and marine algae [33] are being screened for potential lipase inhibitory activity. Ideally, these treatments will act as adjuncts to behavioural and lifestyle changes, aimed at maintenance of weight 
loss and improved health [10]. In this review, we discuss the antiobesity potential of natural inhibitors and analyse their mechanisms on obesity.

\section{Methodology}

A well-known scientific search engines viz. Google Scholar, Pubget Medline PubMed, EMBASE, Mendeley, Science Direct, Scopus, Springer Link were used to retrieve online literature. The results were then cross-referenced to generate a total number of 292 references cited in this review (mostly during the time span of 19752016). The Current review methodically summarises the antiobesity effects of phytochemicals of medicinal plants in various models. Table 1 represents a list of existing medicinal plants/plant extracts/fractions and their effects in PL inhibition in in-vitro and/or in-vivo models. Table 2 represents a list of natural biomaterials showing appetite repressor activity. Table 3 represents existing plant/extracts/bioactive promoting energy expenditure. Table 4 represents plant extracts/bioactive inhibiting adipocyte differentiation. In table 5, natural biomaterials promoting lipid metabolism and acting as anti-obesity agents are shown. In this review, the major biological targets of botanical extracts and isolated compounds are listed with their therapeutic efficacy and possible synergy with other molecules in the extracts is also being discussed.

\section{Factors affecting obesity}

Obesity is a complex disease with multifactorial causes, involving a complex interplay of dietary, lifestyle, environmental and genetic factors.

\section{Age}

Age factor is one of the important criteria involved in causing obesity. As one gets older, one's body's ability to metabolise food slows down, and we require only a few calories to maintain our weight. This is the reason people at $40 \mathrm{y}$ of age tend to gain weight if they eat the same food and do the same activities as they did when they were $20 \mathrm{y}$ old. There is some evidence that when an older woman gives birth, there is a higher risk of obesity in the newborn [34].

\section{Gender}

Women tend to be more overweight than men. Men have a higher resting metabolic rate (means they burn more energy at rest) than women, so men require more calories to maintain their body weight. Additionally, when women become postmenopausal, their metabolic rate decreases. That partly explains why many women gain weight after menopause [35].

\section{Environmental factors}

Environmental factors unknowingly affect our metabolism [36]. It is known that hormones are key regulators in our body metabolism, body weight mechanisms and many pollutants affect our hormones [37]. For convenience, we spend time in temperature controlled rooms [38, 39]. Moreover, smoking also reduces weight [40, 41]. Environmental factors include lifestyle behaviours such as the type of food consumed by a person and his/her day to day activities. These factors unknowingly affect our metabolism systems [42]. Other studies of populations, including migration studies, have shown an increase in average body weight in those who move from a traditional to a westernised environment $[43,44]$.

\section{Psychological factors}

The psychological state of a person also influences eating habits and obesity. Many people eat in response to negative emotions such as boredom, sadness and/or anger. Getting too little sleep can also increase body weight [45-47].

\section{Genetic factors}

Obesity could be viewed as a consequence of the interaction of environmental factors and the individual genetic predisposition. A child of two obese parents has about $80 \%$ chance of becoming obese, whereas the risk is only $15 \%$ for the offspring of two parents of normal weight [48]. People with two copies of the fat mass and obesity (FTO) gene have been found on an average to weigh 3-4 kg more and have a 1.67 folds greater risk of obesity compared to those without the risk allele [49]. Some cases of obesity are related to single-gene mutations, e. g. MC4R gene (melano-cortin-4 receptor) [50], DRD4 gene (dopamine receptor D4) [51], PPAR $\gamma 2$ gene [52] or the leptin genes [53]. Some of the genetic factors predispose us to obesity [54]. There is some evidence that the obese people are more fertile than lean ones [55]. If obesity has a genetic component [56] the percentage of obese people in the population should increase. Moreover, union of obese spouses also promotes obesity in the children [57].

\section{Heredity}

Heredity influences the distribution of fat tissue. Generally, heavy newborns grow into heavy adolescents, more so when either parent is overweight. Moreover, weight regulation in the human body depends upon various genetically determined factors mainly by hormones. Any abnormality in these factors could result in substantial weight gain. Almost $60 \%$ of obese people are said to have inherited this condition. There are several genetic conditions that also contribute or lead to weight gain [58].

\section{Endocrinological causes}

Some people in rare cases are genetically predisposed to obesity due to hormonal imbalance or glandular problems. Cushing syndrome, hypothyroidism, hypogonadism in men and polycystic ovarian syndrome in women, hypothalamic lesions like tumours, infections or severe trauma are some of the genetic causes that lead to obesity [58].

\section{Food intake}

Some patients eat more during the periods of heavy exercise or during pregnancy and are unable to get back to their former eating habits. The increase in obesity can usually be related to the type of food (containing sugar and fat) consumed [59].

\section{Dietary factors}

The world today is more affluent than it ever was; this means that more people have access to a multitude of dietary options. People nowadays are also less active than their predecessors, however, the calorific content of their diet has not decreased; instead, it has increased. Diets around the world have drastically changed; we have transitioned from a high-protein to a high-carbohydrate, high-fat diet. Moreover, the consumption of empty calorie foods like alcohol, aerated drinks, candies, etc. has also been raised sharply. All these coupled with a sedentary lifestyle makes the ideal combination for susceptibility to obesity and diabetes [58].

\section{Sedentary lifestyle}

The rise in obesity is due to a sedentary lifestyle which plays a significant role [60]. There is a large shift toward less physically demanding work worldwide. Currently, at least $60 \%$ of the world's population gets insufficient exercise, due to increased use of mechanised transportation and a greater prevalence of labor-saving technology at home [61]. The WHO indicates people worldwide are taking up less active recreational pursuits. In both children and adults, there is an association between television viewing time and the risk of obesity [62].

\section{Medical and psychiatric illness}

Certain physical and mental illnesses and medication used to treat them can increase the risk of obesity. Medical illnesses that increase obesity risk include several rare genetic syndromes (Crohn's syndrome), as well as some congenital or acquired conditions: hypothyroidism, growth hormone deficiency [63] and eating disorders (binge eating disorder and night eating syndrome)[64]. The risk of overweight and obesity is higher in patients with psychiatric disorders than in persons without psychiatric disorders [65].

\section{Health risks in obesity}

The health risks of overweight and obesity are briefly described below

\section{Hypertension}

Hypertension leads to increase in high blood pressure progressively with higher levels of BMI in men and women [66]. Obesity and 
hypertension are co-morbid risk factors for development of cardiovascular disease. The pathophysiology underlying the development of hypertension associated with obesity includes increased sodium retention and associated increases in vascular resistance, blood volume, increased sympathetic nervous system activity, cardiac output, insulin resistance and suppression of the activity of the rennin-angiotensin-aldosterone system [67-72].

\section{Diabetes mellitus}

Accumulated data demonstrate the association between obesity and non-insulin-dependent diabetes mellitus, which is the most common primary form of diabetes and impaired glucose tolerance. In obese individuals, adipose tissue releases a high amount of non-esterified fatty acids, glycerol, pro-inflammatory cytokines, and hormones. They are linked with the development of insulin resistance, which generates compensatory hyperinsulinemia with overstimulation of pancreatic cells and reduction of insulin receptors [73].

\section{Dyslipidemia}

Obesity is the most common cause of dyslipidemia. Lipid oversupply in a state of obesity, hyperinsulinemia and/or insulin resistance results in increased non-esterified fatty acid availability and, in turn, higher triglyceride (TG) stores in non-adipose tissues, e. g. the muscle, liver, and pancreas [74, 75]. Fatty acid-induced disorders are referred to as lipotoxicity. Thus, elevated TG level is often accompanied by a slight increase in total cholesterol and a marked drop in high-density lipoprotein (HDL) cholesterol. Moreover, lowdensity lipoproteins (LDL) rich in TG, partially metabolised by hepatic lipase, are converted into small LDL, with higher atherogenic potential [76].

\section{Cardiac alterations}

Observation studies have shown that obesity and excess abdominal fat are directly related to cardiovascular risk factors, including high levels of total cholesterol, LDL cholesterol, triglycerides, blood pressure, fibrinogen and insulin [77] and low levels of HDL cholesterol [78], plasminogen activator inhibitor-1 causing impaired fibrinolytic activity is elevated in persons with abdominal obesity [79]. Obesity increases the risk of heart failure, sudden cardiac death, chest pain, and abnormal heart rhythm [80]. Increased electrical alterations in obesity lead to frequent ventricular dysrhythmias even in the absence of heart dysfunction. The annual sudden cardiac death rate was nearly 40 times higher in obese people than in non-obese population $[81,82]$.

\section{Lung disease}

Obesity is associated with an increased risk of chronic respiratory disorders (e. g. asthma, hypoventilation syndrome and sleep apnea). Accordingly, weight loss often leads to symptomatic improvement [83].

\section{Cancer}

The line between diet, obesity and cancer is not completely understood, but the rising worldwide trend in obesity and cancer might be at least in part causal. The putative cause of these obesity-related cancers has been primarily ascribed to excess oestrogen production by the adipose tissue, inflammation due to adipocytokines secreted by adipocytes, infiltrating macrophages or associated stromal cells that might also play an important role $[84,85]$.

\section{Neurological disorders}

Psychological damage caused by overweight and obesity ranges from lowered self-esteem to frank clinical depression. Indeed, rates of anxiety and depression are three to four times higher among obese individuals [86]. Obesity significantly increases the risk of Alzheimer's disease. A strong correlation exists between BMI and high levels of amyloid, i.e. the protein that accumulates in the Alzheimer's brain, destroying nerve cells and producing cognitive and behavioural problems [87].

\section{Clinical management of obesity: drugs and surgery}

Although reduction of caloric intake by diet and increased level of physical activity are very well-known approaches to lose weight, in most cases patients who lose weight, regain the weight in subsequent years suggesting obesity has a high relapse rate making difficulty in treatment. So the treatment has to be a lifelong commitment with proper dietary habits increased physical activities. Many diets have been advocated for weight loss, but there is little scientific evidence to recommend one diet over another [88]. Due to the inconsistent effort in achieving a negative balance through diet and exercise, the needs for drugs and other supplements are fast gaining acceptance. However, drug discovery for anti-obesity agents has long been plagued with inconsistency and side effects. Strategic anti-obesity treatments broadly act through peripherally and centrally. The current scenario in drug discovery for anti-obesity therapeutics mainly focuses on the following mechanisms for energy homoeostasis. 1) Centrally acting: by regulation of food intake 2) Peripherally acting: by affecting absorption of dietary fat, affecting storage and metabolism of fat and/or increasing heat generation from dietary fat [89]. Body weight regulation and energy homeostasis can be viewed as multi-component feedback regulatory mechanisms which provide a vast number of intervening points as targets. In the long term, single point target for body weight management may activate compensatory mechanisms leading to failure of treatment [90].

\section{Currently available synthetic analogues for anti-obesity}

\section{Sibutramine}

Sibutramine (trade names Reductil and Meridia), is an anorectic or appetite suppressant [91]. It is a dual monoamine form, there arenor-adrenaline and serotonin (5-Hydroxytryptamine) reuptake inhibitor [92-94] noradrenaline in the nerve ending of central nervous system(CNS), and this action has anorexigenic and satiety effects, effective in improving metabolic syndrome parameters such as fasting glucose, triglycerides and HDL [95]. The sibutramine is contraindicated in patients with a history of cardiovascular disease, including coronary artery disease, stroke or transient ischemic attack, cardiac arrhythmia, congestive heart failure, peripheral arterial disease, or uncontrolled hypertension [96]. Sibutramine (Meridia) may cause an increase in blood pressure [97].

\section{Orlistat}

The orlistat (Xenical) has intestinal action, acts by inhibiting pancreatic lipase [98], and thus reduction of triglyceride absorption, and its long-term administration accompanying an energy restricted diet, results in weight loss [99]. Compared with sibutramine, the orlistat reduced waist circumference, BMI, systolic and diastolic blood pressure, fasting blood glucose, glycated haemoglobin, total cholesterol and LDL cholesterol in patients with type 2 diabetes mellitus [100]. The common symptoms are a loose stool, the presence of oil in the feces, fecal urgency, fecal incontinence, flatulence and, less frequently abdominal pain and rectal pain [101].

\section{Rimonabant}

Rimonabant's (Acomplia) mode of action in appetite regulation poses involvement of cannabinoid-1-receptors (CB1r) which on stimulation increases demand of food. Rimonabant reduces food intake by blocking CB1r and enhances thermogenesis. Side effects include mood changes, nausea, vomiting, diarrhea, headache, dizziness and anxiety [102].

\section{Lorcaserin}

Lorcaserin, a selective 5-hydroxytryptamine (5-HT2c) receptor agonist developed by Arena Pharmaceuticals, has serotonergic properties and acts as an anorectic. 5-HT2c receptors are located in various parts of the brain, including the hypothalamus, activation of which leads to pro-opiomelanocortin production and results in the weight loss through hypophagia [103]. Fluoxetine and Sertraline have been evaluated in patients with overweight, but without formal indication in the treatment of obesity. Its indication is the treatment of depression and bulimia nervosa [104]. The bupropion is an atypical antidepressant noradrenaline dopamine reuptake inhibitor and a smoking cessation aid; it's also demonstrated decreased food intake and dose-dependent weight loss [105]. Topiramate enhances the activity of gamma aminobutyric acid (GABA); blocks sodium 
channel dependents, antagonises glutamate receptors and inhibits carbonic anhydrase. Secondly, used in epilepsy, mood stabiliser prophylaxis of a migraine and in weight loss [106].

\section{Surgical treatment}

The unsatisfactory results of treatment with diet, exercise or physical, pharmacological therapy reflect the difficulty of the obese patient in changing the lifestyle. With these non-surgical methods often cannot be a significant and sustained weight loss ( $>5 \mathrm{y}$ ), with many recurrences [107]. In 1991, NIH demonstrated safety and the long-term success of several anti-obesity surgeries. Two bariatric surgical procedures have gained popularity for treating obesity [108]. They are gastric bypass surgery and the laparoscopic adjustable gastric band (Lap-Band). Gastric bypass is a permanent surgical option. The surgeon will divide the stomach into two parts and then reconnect the intestine. There are several techniques used to reconnect the intestine, and many gastric bypass surgeries have different names. Gastric bypass surgery causes permanent changes to the stomach and digestive tract and its effects usually cannot be reversed [109].

The laparoscopic adjustable gastric band is an inflatable tube made up of silicone, in the surgery doctor places the band around the top portion of the stomach. The band helps to create a space at the top of the stomach that limits how much food patients can eat at any given time. The band helps a person to feel full and eat less at each meal. Over time, the lap-band patient will lose weight. Doctors recommend the lap-band for patients who have a BMI above 40. Unlike other weight loss surgeries, the lap-band does not permanently alter the digestive tract. Doctors can deflate and remove the band in order to reverse the surgery [109].

\section{Natural inhibitors for treatment of obesity}

Because of dissatisfaction with the high cost and potentially hazardous side effects, a number of researchers demonstrate the potential of natural products and their bioactive compounds for treating obesity. This is currently undergoing exploration of clinical trials and this may be an excellent alternative strategy for developing future effective and safe anti-obesity drugs [110,111]. A vast number of natural products, including crude extracts and isolated bioactive compounds from plants can induce body weight reduction and prevent diet-induced obesity. Based on that, they have been widely used in the treatment of obesity $[112,113]$.

A wealth of information suggested that numerous bioactive components from nature are potentially useful in obesity treatments. The best example is polyphenols and saponins. These show strong anti-obesity activity and include apigenin, catechins and genistein $[114,115]$. A growing body of evidence indicates that natural products having anti-obesity effects can be arranged into five categories based on their distinct mechanisms; they produce (i) decreased lipid absorption, (ii) decreased energy intake, (iii) increased energy expenditure, (iv) decreased pre-adipocyte differentiation and proliferation, (v) decreased lipogenesis and increased lipolysis. Thus, this review emphasises the importance of natural inhibitor along with anti-obesity potential and reviewed the scientific data, including experimental methodologies, bioactive components and its mechanisms of action against obesity.

\section{Inhibitory effect on pancreatic lipase enzyme}

The treatment for obesity makes one of the most important promising criteria in the effort to reduce energy intake through gastrointestinal mechanisms, without altering the central mechanisms, is the development of nutrient digestion and absorption inhibitors [116]. Dietary fat is not directly absorbed by the intestine unless the fat has been subjected to the action of PL. Therefore, PL is one of the most widely studied mechanisms for determining natural products potential efficacy as anti-obesity agents $[116,117]$.

PL is a key enzyme in dietary triacylglycerol absorption, hydrolyzing triacylglycerols to monoacylglycerols and fatty acids. Few natural substances interact directly with the lipases, example orlistat. It is a derivative of naturally occurring lipase inhibitor from Streptomyces toxytricinni [98]. Orlistat lipase inhibition mechanism acts through a covalent bond to the lipase's serine active site [118-120]. Although it is clinically approved for obesity treatment, it has certain unpleasant gastrointestinal side effects $[121,122]$. These side effects result from orlistat include oily spotting, liquid stools, fecal urgency, flatulence, abdominal cramping [123]. Therefore, now researchers are screening novel inhibitors, derived from plants or other natural sources that lack some of these unpleasant side effects [116]. Recent evidence that ethanolic extract of jasmine flowers shown inhibition of PL activity in both in vitro and in vivo [124]. Malaysian researcher screened 32 medicinal plants for anti-lipase activity in vitro assay, in that Eleusine indica (31.36\%), Myristica fragrans (20.23\%), Melastoma candidum (19.67\%) and Phyla nodiflora (18.26\%), respectively showing potential candidates [125], pomegranate leaves [126], Achyranthes aspera L., and Coffea canephora [127], plants possess PL inhibition effects. In 2012, Korean researchers examined 400 plant species, 44 extracts from plants, showed high anti-lipase activity using 2, 4-dinitrophenylbutyrate as a substrate in porcine pancreatic lipase (PPL). Furthermore, 44 plant extracts were investigated for their inhibition of lipid accumulation in 3T3-L1 cells. Among these 44 extracts examined, crude extracts from 4 natural plant species were active. Salicis radicis cortex showed highest fat inhibitory activity; whereas Rubi fructus, Corni fructus and Geranium nepalense exhibited fat inhibitory capacity higher than $30 \%$ at $100 \mu \mathrm{g} / \mathrm{ml}$ in $3 \mathrm{~T} 3-\mathrm{L}$ adipocytes cell lines, suggesting antiobesity activity [128]. Similarly, in 2011 Korean researchers screened 61medicinal plants out of those, Sorbus commixta (stem, leaf) and Viscum album (whole plant) exhibited antilipase activity with IC $\mathrm{C}_{50}$ values of $29.6 \mathrm{mg} / \mathrm{ml}$ and $33.3 \mathrm{mg} / \mathrm{ml}$, respectively [129]. Twenty three medicinal plants were screened which are belonging to 15 different families and compared their PPL effects. Thirteen plants were found to show in vitro inhibitory activities. The nine most active plants have shown an $\mathrm{IC}_{50}$ range of 107.7-342.7 $\mu \mathrm{g} / \mathrm{ml}$. The plants are Anthemis palaestina Boiss. Salvia spinosa L., Ononis natrix L., Fagonia arabica L., Origanum syriaca L., Majorana syriaca (L.) Rafin., Hypericum triquetrifolium Turra, Malva nicaeensis, Chrysanthemum coronarium L., Paronychia argentea Lam [130]. A study was reported on methanolic extracts of 37 traditional Chinese herbal medicines of different families. They were assayed for in vitro activity against PPL activity, among that, Prunella vulgaris L. and Rheum palmatum L. showed significant inhibition [131].

A wide range of plants have possess potent pancreatic lipase inhibition effects, including Panaxjaponicas [132], Platycodi radix [133], Salacia reticulate [134], Nelumbo nucifera [135], and so on described in table 1. This pancreatic lipase inhibitory phytochemicals include mainly, polyphenols, flavonoids, saponins and caffeine [136-139]. Many carbohydrates also possess potent PL inhibitory effects $[140,141]$. But, the effects of these carbohydrates on body weight reduction in animals and humans are controversial [142-150].

Many active metabolites from microorganisms, including lipstatin from Streptomyces toxytricini [151], panclicins from Strepomyces $s p$. NR0619 [152], valilactone and ebelactone from Streptomycesalbolongus [153,154], esterasin from Streptomyces lavendulae [155], caulerpenyne from Caulerpa taxifolia [156], vibralactone from Boreosterem virans [157], and percyguinin from Basidiomycete Stereum Complicatum [158] also possess PL inhibitory activity. Moreover, certain fruiting bodies or mycelia of macrofungi reportedly possess lipase inhibitory activity $[159,160]$.

Some of the most widely studied materials among the many natural sources of PL inhibitions are the different types of tea (e. g green, oolong, and black tea). A significantly different type of tea polyphenols [e. g. L-epicatechin, Epigallocatechin Gallate (ECG), Epigallocatechin gallate (EGG) and (-)-Epigallo-catechin-3-gallate (EGCG)], isolated from tea leaves, showed strong inhibitory activity against PL $[114,161,162]$. These polyphenols require galloyl moieties within their chemical structures and or polymerization of their flavan-3-ols for enhanced PL inhibition [162]. In their search for a PL 54 marine algae were screened. Interestingly, almost all algae showed lipase inhibition activity in either methanol or ethyl acetate extracts [158]. 
Table 1: Plant extracts and isolated compounds showing inhibition on pancreatic lipase

\begin{tabular}{|c|c|c|c|}
\hline Source & Active component & Major activity & References \\
\hline Rubus coreanus Miquel (fruit) & Crude ethanolic extract & $32.5 \pm 1.1 \%$ inhibition & [128] \\
\hline Cornus officinalis Siebold et Zuccarini (fruit) & Crude ethanolic extract & $34.8 \pm 2.3 \%$ inhibition & {$[128]$} \\
\hline Ulmus darvidian for. Suberose (bark) & Crude ethanolic extract & $38.0 \pm 1.9 \%$ inhibition & [128] \\
\hline $\begin{array}{l}\text { Geranium thunbergii Siebold et Zuccarini } \\
\text { (whole grass) }\end{array}$ & Crude ethanolic extract & $31.4 \pm 0.7 \%$ inhibition & [128] \\
\hline Ephedra sinia Stapf. (Herbaceous stem) & Crude ethanolic extract & $25.9 \pm 4.3 \%$ inhibition & {$[131]$} \\
\hline Milettia reticuiata Benth. (Rattan cane) & Crude ethanolic extract & $33.3 \pm 4.2 \%$ inhibition & {$[131]$} \\
\hline $\begin{array}{l}\text { Polygonum cuspidatum Sieb. } \\
\text { (root and rhizome) }\end{array}$ & Crude ethanolic extract & $37.8 \pm 6.5 \%$ inhibition & {$[131]$} \\
\hline Prunella vulgaris L.(ear) & Crude ethanolic extract & $74.7 \pm 10.0 \%$ inhibition & [131] \\
\hline Rheum palmatum L. (root and rhizome) & Crude ethanolic extract & $53.8 \pm 9.0 \%$ inhibition & [131] \\
\hline Salvia miltiorrhiza Bge. (root and rhizome) & Crude ethanolic extract & $32.7 \pm 3.8 \%$ inhibition & {$[131]$} \\
\hline Uncaria macrophylla Wall (aerial part) & Crude ethanolic extract & $30.1 \pm 3.3 \%$ inhibition & [131] \\
\hline $\begin{array}{l}\text { Juniperus communis(bark) and Illicium } \\
\text { religiosum (wood) }\end{array}$ & Crude ethanol/water extract & $\begin{array}{l}\mathrm{IC}_{50}=20.4 \text { and } 21.9 \mu \mathrm{g} / \mathrm{ml} \text {, } \\
\text { respectively }\end{array}$ & [136] \\
\hline Panax japonicas (rhizomes) & Chikusetsusaponins & $22 \%$ decrease in body weight gain & {$[132]$} \\
\hline Carica papaya $\mathrm{L}$. & Crude aqueous extract & $55.77 \pm 0.4 \%$ inhibition & [159] \\
\hline Thea sinesis (oolong tea) & Crude aqueous extract (caffeine) & $10 \%$ decrease in body weight gain & [163] \\
\hline Platycodi radix & Platycodin saponins & $13 \%$ decrease in body weight gain & [164-167] \\
\hline Platycodi radix & $\begin{array}{l}\text { Crude aqueous/ethanolic extract } \\
\text { (saponin) }\end{array}$ & $12 \%$ decrease in body weight gain & [133] \\
\hline Acanthopanax santicosus & $10.6 \%$ ellagic acid & $54 \%$ decrease in body weight gain & [168] \\
\hline Cassia mimosoides & Proanthocyanidin & $\begin{array}{l}\mathrm{IC}_{50}=0.11 \mathrm{mg} / \mathrm{ml} ; 60 \% \text { decrease in } \\
\text { body weight gain }\end{array}$ & [169] \\
\hline Kochia scoparia (fruits) & Crude aqueous extract (saponins) & $19 \%$ decrease in body weight gain & [137] \\
\hline $\begin{array}{l}\text { Afromomum meleguetta and } \\
\text { Spilanthes acmella }\end{array}$ & Crude ethanolic extract & $\begin{array}{l}90 \%, 40 \% \text { lipase inhibition } \\
\text { respectively }\end{array}$ & {$[170]$} \\
\hline Salacia reticulate (mixed with cyclodextrin) & Crude aqueous extract & $27 \%$ decrease in body weight gain & [133] \\
\hline Thea sinensis (leaf) & Saponins & $17 \%$ decrease in body weight gain & {$[163,171]$} \\
\hline Nelumbo nucifera (leaf) & Crude ethanolic extract & $28 \%$ decrease in body weight gain & [135] \\
\hline Trigonella foenum graecum L. (seed) & Crude ethanolic extract & $14 \%$ decrease in body weight gain & {$[172]$} \\
\hline Salix matsudana (leaf) & $\begin{array}{l}\text { Poly phenol (PP), } \\
\text { Flavonoid glucoside }\end{array}$ & $\begin{array}{l}20 \% \text { decrease in body weight gain } \\
19 \% \text { decrease in body weight gain }\end{array}$ & {$[173,174]$} \\
\hline Vitis vinifera & Crude ethanolic extract & $\begin{array}{l}\text { Inhibitory effect on lipase activity = } \\
80 \%\end{array}$ & [10] \\
\hline Eriochloa villosa & Crude methanolic extract & $\begin{array}{l}\text { Inhibitory effect on lipase activity = } \\
83 \%\end{array}$ & {$[175]$} \\
\hline Orixa japonica & Crude methanolic extract & $\begin{array}{l}\text { Inhibitory effect on lipase activity = } \\
81 \%\end{array}$ & [175] \\
\hline Setariia italic & Crude methanolic extract & $\begin{array}{l}\text { Inhibitory effect on lipase activity = } \\
80 \%\end{array}$ & [175] \\
\hline Salvia officinalis L. & Methanolic extract (carnosic acid) & $\mathrm{IC}_{50}=36 \mu \mathrm{g} / \mathrm{ml}$ & [176] \\
\hline Scabiose tschiliesis Gun. & Triterpenoid saponins & $\begin{array}{l}\text { Maximum activity: almost } 100 \% \\
\text { with prosapogenin 1B (1 mg/ml) }\end{array}$ & [177] \\
\hline Acanthopanax sessiliflorous & Lupane-type saponins & $40 \%$ decrease in body weight gain & [178] \\
\hline Aesoulus turbinate (seed) & Escin & $\mathrm{IC}_{50}=14 \mu \mathrm{g} / \mathrm{ml}$ with escinIIb & [179] \\
\hline Cyclocarya paliurus (Batal) IIinskaja & Crude aqueous extract & $\mathrm{IC}_{50}=9.1 \mu \mathrm{g} / \mathrm{ml}$ & {$[180]$} \\
\hline Ziziphus Mauritiana & Crude extract & $68 \%$ decrease in body weight gain & [181] \\
\hline Gardenia jasminoides (fructus) & Croci, crocetin & $\begin{array}{l}\mathrm{IC}_{50}=2.1 \mathrm{mg} / \mathrm{ml} \text { with crocetin; } 25 \% \\
\text { decrease in body weight gain with } \\
\text { crocin }\end{array}$ & {$[182,183]$} \\
\hline Dioscorea nipponica & Crude methanolic extract & $\begin{array}{l}\mathrm{IC}_{50}=5-10 \mu \mathrm{g} / \mathrm{ml}, 37 \% \text { decrease in } \\
\text { body weight gain }\end{array}$ & [184] \\
\hline Coffea canephora & $\begin{array}{l}\text { Caffeine, chlorogenic acid, } \\
\text { neochlorogenic acid, feruloyquinic acids }\end{array}$ & $157 \%$ decrease in body weight gain & [159] \\
\hline Glycyrrhiza uralensis & Licochalcone A & $\mathrm{IC}_{50}=35 \mu \mathrm{g} / \mathrm{ml}, \mathrm{K}_{\mathrm{i}}=11.2 \mu \mathrm{g} / \mathrm{ml}$ & [185] \\
\hline $\begin{array}{l}\text { Spirulina platensis } \\
\text { (marine algae) }\end{array}$ & Crude methanolic extract & $55 \pm 0.23 \%$ inhibition & {$[186]$} \\
\hline Gracilaria corticata (marine algae) & Crude methanolic extract & $45 \pm 0.21 \%$ inhibition & [186] \\
\hline Fungus, Leetiporus sulphureus & Mycelia extract & $\begin{array}{l}\text { Inhibitory effect on lipase activity = } \\
83 \%\end{array}$ & [187] \\
\hline Fungus, Tylopilus felleus & Mycelia extract & $\begin{array}{l}\text { Inhibitory effect on lipase activity = } \\
96 \%\end{array}$ & [187] \\
\hline Fungus, Hygrocybe conica & Mycelia extract & $\begin{array}{l}\text { Inhibitory effect on lipase activity = } \\
97 \%\end{array}$ & [187] \\
\hline Streptomyces toxytricini & Lipistatin & $\mathrm{IC}_{50}=0.14 \mu \mathrm{g} / \mathrm{mol}$ & [27] \\
\hline Actinomycetes sp. & Valilactone & $\mathrm{IC}_{50}=0.00014 \mu \mathrm{g} / \mathrm{ml}$ & [153] \\
\hline Citrus unshiu & Hesperidin & $\mathrm{IC}_{50}=32 \mu \mathrm{g} / \mathrm{ml}$ & [188] \\
\hline
\end{tabular}




\section{Suppressive effect on food intake}

For the past30 y, Sibutramine is the first new drug approved by FDA for treating obesity via appetite suppression [91]. Its main mechanism involves an increase in the feeling of satiety by controlling noradrenalin, serotonin/5-hydroxytryptamine, and dopamine $[189,190]$. However, sibutramine shown some known side effects, including dry mouth, constipation, and insomnia [143]. Body weight regulation through appetite control is multifactorial events resulting from neurological and hormonal interrelationships. A line of evidence indicates that dopamine, histamine, serotonin, and their associated receptor activities are closely connected with satiety regulation. These receptors may enable researchers to target better their searches for drugs that treat obesity through energy intake reduction [191]. Molecules that act via peripheral satiety peptide systems alter the various hypothalamic neuropeptide levels. Also, they change the main CNS appetite monoamine neurotransmitter levels and they may be the suitable candidates for appetite suppressant $[192,193]$.

Appetite suppressants control hunger centres in the brain, providing a satiety signal. However, ghrelin secretion in the stomach may increase with decreased food intake, stimulating more food intake. Therefore, ghrelin antagonism may decrease the appetite that potentially occurs with decreased feeding, thus, may be a potential adjunctive treatment for obesity. An example of a natural appetite suppressant is Hoodia gordonii. It regulates appetite and significantly reduces calorie intake and boosts weight loss [194].
Natural (_)-hydroxy citric acid (HCA) from Garcinia cambogia, is a potential natural appetite suppressant. It is available under the names HCA-SX and Super CitriMax [195]. HCA is a competitive inhibitor of adenosine 5-triphosphate-citrate lyase, leading to a decreased acetyl coenzyme A production and decreased fatty acid synthesis. HCA is also thought to suppress food intake via loss of appetite by stimulating liver gluconeogenesis. HCA is also reported to act by increasing the availability of 5-hydroxytryptamine or serotonin, which is a neurotransmitter implicated in regulating eating behaviour and appetite control. The central metabolism of glucose also suppresses food intake, mediated by the hypothalamic Adenosine monophosphate-activated protein kinase activation (AMPK)/malonyl-CoA signalling system [196]. Central administration of glucose increases hypothalamic malonyl-CoA decreases orexigenic neuropeptide expression and suppresses food intake. Centrally-administered fructose provokes feeding, via the AMPK/malonyl-CoA signalling pathway. Thus, decoctions prepared from natural sources containing excessively high fructose levels may suppress the hypothalamic malonyl-CoA signalling pathway, thereby exerting an orexigenic effect [197]. Hypericum perforatum increases the serotonin quantity present within synaptosomes by inhibiting synaptosomal uptake of serotonin, which suppresses the appetite and reduces food intake. Thus increased serotogenic transmission might be the link between antidepressant and anti-obesity activities of H. perforatum [194]. Some natural appetite suppressants are listed in table 2.

Table 2: Anti-obesity biomaterials showing appetite-repression activity

\begin{tabular}{|c|c|c|}
\hline Source & Used part and/or active constituent & References \\
\hline Caralluma fimbriata & Total dry extract & [198] \\
\hline Panax ginseng (root) & Crude saponins & [199] \\
\hline Terminalia paniculat Roth.(bark) & Crude ethanolic extract & [200] \\
\hline \multicolumn{3}{|l|}{ Family: Combretaceae } \\
\hline Bauhinia variegate & Crude aqueous extract & [201] \\
\hline \multicolumn{3}{|l|}{ Bauhinia variegate (root) } \\
\hline Pinellia ternate & Crude aqueous extract & [202] \\
\hline Garcinia cambogia & $(-)-\mathrm{HCA}$ & [203] \\
\hline Camellia sinensis (leaf) & $(-)$-EGCG & [204] \\
\hline Hoodia gordonii and $H$. pilifera & Steroidal glycoside (P57AS3) & {$[205,206]$} \\
\hline Phaseolus vulgaris and Robinia pseudoacacia & Lectins & [207] \\
\hline Pinus koraienis(pine nut) & Pine nut fatty acids & [208] \\
\hline Ephedra species & Ephedrine & [209] \\
\hline Citrus aurantum & Synephrine & [210] \\
\hline Hypericum perforatum & Total extract & [211] \\
\hline Nelumbo nucifera (leaf) & Crude ethanolic extract (flavonoid) & [212] \\
\hline Panax ginseng(berry) & Crude ethanolic extract & [213] \\
\hline Glycine max (soybean) & b-conglycinin,glycinin(globulins) & {$[214,215]$} \\
\hline Undaria pinnatifida(sea weed) & Fucoxanthin & [216-218] \\
\hline Undaria pinnatifida (seaweed) & $\begin{array}{l}\text { Eicosapentaenoic acid (EPA) and Docosahexaenoic } \\
\text { acid (DHA) }\end{array}$ & [219] \\
\hline Sterculic foetida (seeds) & Petroleum ether extract & [220] \\
\hline Caralluma fimbriata & Total dry extract & [198] \\
\hline Panax ginseng (root) & Crude saponins & [199] \\
\hline Terminalia paniculat Roth.(bark) & Crude ethanolic extract & {$[200]$} \\
\hline \multicolumn{3}{|l|}{ Family: Combretaceae } \\
\hline Bauhinia variegate & Crude aqueous extract & [201] \\
\hline \multicolumn{3}{|l|}{ Bauhinia variegate (root) } \\
\hline Pinellia ternate & Crude aqueous extract & [202] \\
\hline Garcinia cambogia & $(-)-\mathrm{HCA}$ & [203] \\
\hline Camellia sinensis (leaf) & $(-)$-EGCG & [204] \\
\hline Hoodia gordonii and H. pilifera & Steroidal glycoside (P57AS3) & {$[205,206]$} \\
\hline Phaseolus vulgaris and Robinia pseudoacacia & Lectins & [207] \\
\hline Pinus koraienis(pine nut) & Pine nut fatty acids & [208] \\
\hline Ephedra species & Ephedrine & [209] \\
\hline Citrus aurantum & Synephrine & [210] \\
\hline Hypericum perforatum & Total extract & [211] \\
\hline Nelumbo nucifera (leaf) & Crude ethanolic extract (flavonoid) & [212] \\
\hline Panax ginseng(berry) & Crude ethanolic extract & [213] \\
\hline Glycine max (soybean) & b-conglycinin, glycinin(globulins) & {$[214,215]$} \\
\hline Undaria pinnatifida(sea weed) & Fucoxanthin & [216-218] \\
\hline Undaria pinnatifida (seaweed) & EPA and DHA & [219] \\
\hline Sterculic foetida (seeds) & Petroleum ether extract & [220] \\
\hline
\end{tabular}




\section{Stimulatory effects on energy expenditure}

Stimulation of energy expenditure is one of the important cretiria in obesity. Results of recent experiments showed that natural inhibitors active against energy expenditure stimulants are more effective. Excessive adiposity results from an imbalance in energy homoeostasis, in which the consequences of excessive food intake are not balanced by increased energy expenditure [221, 222]. Energy expenditure components can be separated into a number of different categories. The simplest scheme divides energy expenditure into three categories: (1) physical activity (2) obligatory energy expenditure, and (3) adaptive thermogenesis. To regulate body weight and energy expenditure, mammalian brown adipocyte tissue (BAT) establishes non-shivering thermogenesis, through dissipation of excess energy as heat [223]. BAT plays an important role in obesity control by controlling energy balance through uncoupling protein (UCP1) responsible for oxidative phosphorylation. Thus, searching for substances that up regulate UCP1 gene expression may be a worth strategy for achieving obesity control through increased energy expenditure [224]. An example is the ethanolic extract of Solanum tuberosum, which activated the expression of UCP3 in BAT and liver which significantly reduced fat weight in HFD fed rats [225]. Many natural compounds have been proposed as treatments for obesity via enhanced energy expenditure including caffeine, capsaicin, green tea and its extracts [112]. Some natural appetite suppressants are listed in table 3.

Table 3: Anti-obesity plant extracts and bio-molecules promoting energy expenditure

\begin{tabular}{|c|c|c|c|}
\hline Source & Active component & Major activity & References \\
\hline Pinellia ternate & Crude aqueous extract & Slight decrease in body weight gain & {$[202]$} \\
\hline Nelumbo nucifera (leaf) & Crude ethanolic extract & $15 \%$ decrease in body weight gain & {$[114,191,212]$} \\
\hline Camellia sinensis & EGCG & $8 \%$ decrease in body weight & {$[114,191,227]$} \\
\hline Panax ginseng (berry) & Crude ethanolic extract & $13 \%$ decrease in body weight gain & [213] \\
\hline Glycine max (soybean) & $\beta$-conglycinin, glycinin (globulins) & $10 \%$ decrease in body weight gain & {$[214,215]$} \\
\hline Undariapinnatifida (sea weed) & Fucoxanthin & $17 \%$ decrease in body weight gain & {$[217,218]$} \\
\hline
\end{tabular}

\section{Inhibitory effect on adipocyte differentiation}

Adipocyte plays a central role in the maintenance of lipid homoeostasis and energy balance, by storing triglycerides and releasing free fatty acids in response to changing energy demands. Because adipocyte tissue growth can be due to both hyperplasia and hypertrophy of adipocytes. Several studies screening for anti-obesity materials have focused on the processes of adipocyte proliferation and differentiation [226]. In this search, 3T3-L1 preadipocyte cells are currently used as an in vitro model for the study of obesity, because such cells accumulate triglycerides upon differentiating in culture [227, 228]. This is due to the expression of adipocyte-specific genes, such as PPARc and enhancer binding protein $[229,230]$. For this reason, natural products that specifically target adipogenesis inhibition had been considered promising with regard to their potential in the treatment of obesity. However, current research suggests that inhibiting adipogenesis or adipose tissue expansion is unhealthy, leading to type 2 diabetes and other metabolic diseases, such as atherosclerosis [229].
Fatty acids, particularly polyunsaturated fatty acid (PUFA), acts as signal transducing molecules in adipocyte differentiation. In adipocyte tissue, saturated and monounsaturated fatty acids are more readily acylated into triglycerides than PUFA [231-233]. Thus, PUFA plays a central role in suppressing fatty acid synthesis and regulating adipocyte differentiation through suppression of late phase adipocyte differentiation [233, 234]. Recent reports have demonstrated another interesting mechanism, in the extract of macro fungus Cordyceps militaris mycelia, which suppressed 3T3-L1 adipocyte differentiation through activation of the aryl hydrocarbon receptor [235]. The wide variety of natural products found to inhibit preadipocyte proliferation and/or the apoptotic effect. In addition, to showing inhibitory activity against adipocyte differentiation, several naturally occurring compounds have displayed apoptotic effects on maturing preadipocytes (esculetin, resveratrol, guercetin, genistein, EGCG, capsaicin, and conjugated linoleic acids). Examples of some natural products and isolated biomolecules with adipocyte differentiation inhibitory effects are given in table 4 .

Table 4: Anti-obesity plant extracts and bio-molecules inhibiting adipocyte differentiation

\begin{tabular}{|c|c|c|}
\hline Source & Active component & References \\
\hline Garcinia cambogia & $(-)-\mathrm{HCA}$ & {$[236]$} \\
\hline Cassia auriculata(leaf) & Crude aqueous extract & [237] \\
\hline Pinusdensiflora & Crude aqueous extract & [238] \\
\hline Corsrhizometidi & Berberine & [241] \\
\hline Chili pepper (Capsicum) & Capsaicin & [242] \\
\hline LagerstroemiaSpeciosa L. (banana leaf) & Hot water extract (tannic acid) & [246] \\
\hline Undariapinnatifida (brown algae) & Fucoxanthin & [247] \\
\hline Camellia sinensis(green tea) & $(-)-E C G$ & {$[248-250]$} \\
\hline Lithospermumerythrorhizon & Shikonin & {$[251]$} \\
\hline Zizyphus jujube (fruit) & Extract of chloroform Fraction & {$[252]$} \\
\hline Garlic & Ajoene & [253] \\
\hline Humuluslupulus & Xanthohumol & {$[254,255]$} \\
\hline Lagerstroemia speciosa(leaf) & Ellagitannins & [246] \\
\hline AscophyllumNodosum & Aqueous methanolic extract & [256] \\
\hline Wasabia japonica (leaf) & Hot water extract & {$[257]$} \\
\hline Coriolous versicolor (mushroom fruit body) & $(-)$ Ternatin & [258] \\
\hline Cordycepsmilitaris & Mycelial extract & {$[194]$} \\
\hline Ipomoea batatas(root) & Sporamin & [259] \\
\hline Rosmarinusofficinalis & Carnosic & {$[260]$} \\
\hline Curcuma longa & Curcumin & [261-263] \\
\hline Linumusitatissimum (flax seed) & (-)Secoisolariciresinol & {$[264]$} \\
\hline Hibiscus sabdariffa & Flower extract & {$[265]$} \\
\hline Solanum tuberosum & Ethanolic extract & {$[225]$} \\
\hline Soy isoflavone & Genistein & {$[266]$} \\
\hline Undariapinnatifida & Neoxanthin & [267] \\
\hline Commiphoramukul & Cis-guggulsterone & [268] \\
\hline Rehmanniaglutinosa & Crude ethanolic extract & [269] \\
\hline Eriobotrta japonica & Corosolic acid & {$[270]$} \\
\hline Irvingiagabonesis(seed) & Extract & [271] \\
\hline Terminalia paniculatRoth.(bark) Family: Combretaceae & Crude ethanolic extract & {$[200]$} \\
\hline
\end{tabular}




\section{Regulatory effects on lipid metabolism}

The pharmacological targeting of lipolysis can be achieved by stimulating triglyceride hydrolysis in order to diminish fat stores, thereby combating obesity. This option requires the associated oxidation of the newly released fatty acids which led to the development of the b3-adrenergic agonists [272]. The flavonoids from Nelumbo nucifera leaves are examples of the biomaterial inhibitors involved in b3-adrenergic receptor activation [212]. The activity of oolong tea is a good example of another anti-obesity mechanism. Caffeine, one of the major bioactive components in oolong tea, possesses both a positive charge and a hydrophobic area like that of adrenaline. Caffeine's mechanism of lipolytic action might be due to its binding to the phospholipid phosphate groups and the subsequent interactions between the lipase and triglyceride portions of lipid droplets, eliciting lipolysis [163]. Examples of some natural products with adipocyte differentiation inhibitory effects are given in table 5.

Table 5: Anti-obesity biomaterials promoting lipid metabolism

\begin{tabular}{|c|c|c|c|c|}
\hline Source & Active component & Major activity & Mechanism of action & References \\
\hline Salacia oblonga (root) & Mangiferin & $40 \%$ decrease in liver/body weight ratio & Hepatic PPARa activator & {$[273,274]$} \\
\hline Lex paraguariensis & Crude water extract & $11 \%$ decrease in body weight gain & $\begin{array}{l}\text { Downregulation of adipose } \\
\text { tissue genes }\end{array}$ & {$[275]$} \\
\hline $\begin{array}{l}\text { Mixture of Morus alba, } \\
\text { Melissa officinalis, } \\
\text { Artemisia (leaf) }\end{array}$ & Crude aqueous extract & $7 \%$ decrease in body weight gain & Hepatic PPARa activator & [276] \\
\hline Cortidis Rhizome & Berberine & $13 \%$ decrease in body weight gain & AMPK & {$[277]$} \\
\hline Nelumbo nucifera (leaf) & $\begin{array}{l}\text { Crude ethanolic extract } \\
\text { (flavonoid) }\end{array}$ & $15 \%$ decrease in body weight gain & $\begin{array}{l}\text { Activation of } \beta \text {-adrenegic } \\
\text { receptor }\end{array}$ & [212] \\
\hline Curcuma longa L. & $\begin{array}{l}\text { Curcumin } \\
\text { Curcuminoids }\end{array}$ & $\begin{array}{l}7 \% \text { decrease in body weight gain } \\
11 \% \text { decrease in body weight gain }\end{array}$ & $\begin{array}{l}\text { Reversal of inflammatory } \\
\text { and metabolic derangement } \\
\text { Alteration in fatty acid } \\
\text { metabolism }\end{array}$ & {$[278-280]$} \\
\hline $\begin{array}{l}\text { Eucommia } \\
\text { Ulmoides(leaf) }\end{array}$ & Crude aqueous extract & No data & $\begin{array}{l}\text { Downregulation of lipogenic } \\
\text { enzymes }\end{array}$ & [281] \\
\hline $\begin{array}{l}\text { Coixlachrymajobi var. } \\
\text { mayeun (seed) }\end{array}$ & Crude aqueous extract & $36 \%$ decrease in body weight gain & $\begin{array}{l}\text { Modulation of lepin and } \\
\text { tumour necrosis factor (TNF- } \\
\alpha)\end{array}$ & {$[282]$} \\
\hline Salacia reticulate (root) & $\begin{array}{l}\text { Aqueous extract } \\
\text { (polyphenolic compounds) }\end{array}$ & $7 \%$ decrease in body weight gain & $\begin{array}{l}\text { Inhibition of lipid } \\
\text { metabolising enzymes and } \\
\text { stimulation of lipolysis }\end{array}$ & [283] \\
\hline $\begin{array}{l}\text { Glycyrrhiza glabra } \\
\text { L.(root) }\end{array}$ & Licorice flavonoid oil & $30 \%$ decrease in body weight gain & PPAR $\gamma$ agonistic activity & [284] \\
\hline Diospyros kaki (leaf) & Crude methanolic extract & $11 \%$ decrease in body weight gain & $\begin{array}{l}\text { Modulation of leptin and } \\
\text { lipogenic enzymes }\end{array}$ & [277] \\
\hline Morus alba L.(leaf) & Crude aqueous extract & $7 \%$ decrease in body weight gain & PPARs agonistic activity & {$[110]$} \\
\hline Panax ginseng & Crude aqueous extract & $8 \%$ decrease in body weight gain & PPARs agonistic activity & [110] \\
\hline $\begin{array}{l}\text { Lagestroemia speciosa } \mathrm{L} . \\
\text { (leaf) }\end{array}$ & Crude aqueous extract & $3 \%$ decrease in body weight gain & PPARs agonistic activity & {$[110]$} \\
\hline Glycyrrhizauralensis & $\begin{array}{l}\text { Crude ethanolic extract } \\
\text { (flavonoid) }\end{array}$ & $22 \%$ decrease in body weight gain & PPAR $\gamma$ agonistic activity & [285] \\
\hline Evodia ruraecarpa(fruit) & $\begin{array}{l}\text { Crude ethanolic extract } \\
\text { (evodiamine) }\end{array}$ & $23 \%$ decrease in body weight gain & $\begin{array}{l}\text { Vanilloid receptor agonistic } \\
\text { activity }\end{array}$ & [286] \\
\hline $\begin{array}{l}\text { RhynchosiaVolubilis } \\
\text { (black soybean) }\end{array}$ & Tripepide (Ile-Gln-Asn) & $\mathrm{IC}_{50}=0.014 \mathrm{mg}$ protein $/ \mathrm{mol}$ & AMPK activation & [287] \\
\hline Soybean & $\begin{array}{l}\text { Genistein+L-carnitine } \\
\text { (soy isoflavone) }\end{array}$ & $254 \%$ decrease in body weight gain & PPARs agonistic activity & [288] \\
\hline Coffea canephora & $\begin{array}{l}\text { Caffeine, chlorogenic acid, } \\
\text { neochlorogenic acid, feruloyl } \\
\text { quinic acids }\end{array}$ & $157 \%$ decrease in body weight gain & $\begin{array}{l}\text { Inhibition of fat absorption, } \\
\text { activation of fat metabolism }\end{array}$ & [159] \\
\hline $\begin{array}{l}\text { Glycine max (Soy) } \\
\text { Isoflavone }\end{array}$ & Genistein & $\begin{array}{l}90 \% \text { inhibition of adipocyte } \\
\text { differentiation, } 43 \% \text { decrease in cell } \\
\text { adipocyte viability }\end{array}$ & $\begin{array}{l}\text { AMPK activation, } \\
\text { Adipocyte apoptosis }\end{array}$ & {$[270]$} \\
\hline $\begin{array}{l}\text { Rubus idaeus } \\
\text { (rasberry) }\end{array}$ & $\begin{array}{l}\text { 4-(4-Hydroxyphenyl) } \\
\text { Butan-2-one (RK) }\end{array}$ & $17 \%$ decrease in body weight gain & Increase lipolysis & [289] \\
\hline Solanum tuberosum & Crude extract & $5 \%$ decrease in body weight gain & $\begin{array}{l}\text { Downregulation of P38 MAPK } \\
\text { and Upregulation of UCP3 }\end{array}$ & {$[225]$} \\
\hline Momordica charantia & Crude ethanolic extract & $\begin{array}{l}61 \% \text { increase in glucose uptake with } \\
0.5 \text { nmol insulin and } 75 \% \text { increase in } \\
\text { adiponectin secretion }\end{array}$ & $\begin{array}{l}\text { Enhance glucose uptake and } \\
\text { adiponectin secretion }\end{array}$ & [290] \\
\hline Toona sinensis (leaf) & Crude ethanolic extract & $\begin{array}{l}156 \% \text { increase in glycerol release } \\
\text { Lipolytic activity }\end{array}$ & Lipolytic activity & [291] \\
\hline Cinnamoni cassia & Cinnamon & 3.1 fold increase in PPAR $\gamma$ levels & PPAR $\gamma$ agonistic activity & [292] \\
\hline
\end{tabular}

\section{Conclusion and future prospects}

Body weight management is a lifelong process, and permanent weight reduction is difficult to achieve. The ultimate cause of obesity is an imbalance between calorie intake and energy expenditure resulting from complex interactions between many genetic and environmental factors. Obesity is multifactorial and the chronological disease that affects millions of people worldwide and contributes substantial morbidity and mortality. Anti-obesity pharmacological treatment should be administered only when BMI above 25 and considered safe and effective for the obese subject.

Over the past $30 \mathrm{y}$, few obesity treatment drugs have been developed or approved. Only two drugs are currently available, and 
some drugs have been withdrawn from the market due to its serious side effects. Sibutramine and orlistat may cause weight loss of up to $10 \%$ when used in combination with dieatary, behavioural and exercise therapy. Natural bio-molecules, inhibitors or products can play a safe and effective role in obesity especially those containing fibres, polyphenols, alkaloids and sterols. Natural biomaterials with potential action on causative metabolic pathways of obesity work without affecting CNS. The need exists for anti-obesity drugs having greater effectiveness, which are better tolerated. In the future, the active exploration of many natural sources may provide hope for new developments based on a growing understanding of the complex mechanism involved in body fat content regulation. Ideally, such exploration and research will lead to a safer and more effective pharmacological treatment for obesity.

\section{ACKNOWLEDGEMENT}

Authors are grateful to Mangalore University for the support and encouragement.

\section{CONFLICTS OF INTERESTS}

Declared none

\section{REFERENCES}

1. Singla P, Bardoloi A, Parkash AA. Metabolic effects of obesity: a review. World J Diabetes 2010;1:76-88.

2. Derdemezis CS, Voulgari PV, Drosos AA, Kiortsis DN. Obesity, adipose tissue and rheumatoid arthritis: coincidence complex relationship? Clin Exp Rheumatol 2011;29:712-27.

3. Kumanyika SK, Obarzanek E, Stettler N, Bell R, Field AE, Fortmann SP, et al. Population-based prevention of obesity: the need for comprehensive promotion of healthful eating, physical activity, and energy balance: a scientific statement from American Heart Association Council on Epidemiology and Prevention, Interdisciplinary Committee for Prevention (formerly the expert panel on population and prevention science). Circulation 2008;118:428-64.

4. World Health Organization. Obesity; 2014. Available from: http://www.who.int/mediacentre/factsheets/fs311/en/. [Last accessed on 14 Aug 2016].

5. Withrow D, Alter DA. The economic burden of obesity worldwide: a systematic reviewing of the direct costs of obesity. Obes Rev 2011;12:131-41.

6. Schrauwen P, Westerterp KR. The role of high-fat diets and physical activity in the regulation of body weight. Br J Nutr 2000;84:417-27.

7. Abete I, Astrup A, MartnezJA, Thorsdottir I, Zulet M. Obesity and the metabolic syndrome: the role of different dietary macronutrient distribution patterns and specific nutritional components on weight loss and maintenance. Nutr Rev 2010;68:214-31.

8. Little T, Horowitz M, Feinle-Bisset C. Modulation by high-fat diets of gastrointestinal function and hormones associated with the regulation of energy intake: implications for the pathophysiology of obesity. Am J Clin Nutr 2007;86:531-41.

9. Rubio M, Gargallo M, Millán A, Moreno B. Drugs in the treatment of obesity: sibutramine, orlistat and rimonabant. Public Health Nutr 2007;10:1200-5.

10. Moreno D, Ilic N, Poulev A, Brasaemle D, Fried S, Raskin I. Inhibitory effects of grape seed extract on lipases. Nutrition 2003;19:876-79.

11. Yun JW. Possible anti-obesity therapeutics from the nature-a review. Phytochemistry 2010;71:1625-41.

12. Sumantran V. Experimental approaches for studying uptake and action of herbal medicines. Phytother Res 2007;21:210-4.

13. Verma RK, Paraidathathu T. Herbal medicines used in the traditional Indian medicinal system as a therapeutic treatment option for overweight and obesity management: a review. Int J Pharm Pharm Sci 2014;6:40-7.

14. Naquvi KJ, Ansari SH, Najmi AK. Quality standards of safoof-emuhazzil, an anti-obesity unani formulation. Int J Pharm Pharm Sci 2013;5:513-8.

15. Patel R, Patel J, Kakkar S. Formulation and development of antiobesity liquid formulation containing Garcinia cambogia extract, l-carnitine and chromium picolinate. Indo Global J Pharm Sci 2013;3:40-51.

16. Nazish I, Anasari SH, Arora P. Standardization of polyherbal formulation vyosadiguggulu for obesity. Int J Pharm Pharm Sci 2012;4:177-80.

17. Chandrasekaran CV, Vijayalakshmi MA, Prakash K, Bansal VS, Meenakshi J, Amit A. Review article: an herbal approach for obesity management. Am J Plant Sci 2012;3:1003-14.

18. Ortinau LC, Nickelson KJ, Stromsdorfer KL, Naik CY, Pickering RT, Haynes RA, Fritsche KL, et al. Sterculic oil, a natural inhibitor of scd1, improves the metabolic state of obese oletf rats. Obesity 2013;21:344-52.

19. Fayeza MD, SathishKumar SN, Kim YJ, Kim SY, Yang DC. Ginsenoside F2 possesses anti-obesity activity via binding with PPAR $\gamma$ and inhibiting adipocyte differentiation in the 3T3-L1 cell line. J Enzyme Inhib Med Chem 2015;30:9-14.

20. Fayeza MD, SathishKumar SN, Kim YJ, Kim SY, Yang DC. In silico screening of ginsenoside Rh1 with PPAR $\gamma$ and in vitro analysis on 3T3-L1 cell line. J Mol Simulation 2015;41:1219-26.

21. Park KS. Raspberry ketone, a naturally occurring phenolic compound, inhibits adipogenic and lipogenic gene expression in 3T3-L1 adipocytes. J Pharm Biol 2015;53:870-5.

22. Athesh K, Divakar M, Brindha P. Anti-obesity potential of Cyperus rotundus L. aqueous tuber extract in rats fed on highfat cafeteria diet. Asian J Pharm Clin Res 2014;7:88-92.

23. Ahmed HH, Hamza AH, Kotob SK. Corn silk offers multimechanistic approaches in mitigating obesity in rodents. Asian J Pharm Clin 2016;9:292-8.

24. Bray G, Ryan D. Drug treatment of the overweight patient. Gastroenterology 2007;132:2239-52.

25. Gonzalez-Castejon $\mathrm{M}$, Rodriguez-Casado A. Dietary phytochemicals and their potential effects on obesity: a review. Pharmacol Res 2011;64:438-55.

26. McClendon K, Riche D, Uwaifo G. Orlistat: current status in clinical therapeutics. Expert Opin Drug Saf 2009;8:727-44.

27. Weibel EK, Hadvary P, Hochuli E, Kupfer E, Lengsfeld H. Lipstatin, an inhibitor of pancreatic lipase, produced by Streptomyces tox tricine I. Producing organism, fermentation, isolation and biological activity. J Antibiot 1987;40:1081-5.

28. Viner RM, Hsia Y, Tomsic T, Wong CK. Efficacy and safety of anti-obesity drugs in children and adolescents: systematic review and meta-analysis. Obes Rev 2010;11:593-602.

29. Heymsfield SB, Segal KR, Hauptman J, Lucas CP, Boldrin MN, Rissanen A. Effects of weight loss with orlistat on glucose tolerance and progression to type 2 diabetes in obese adults. Arch Int Med 2000;160:1321-6.

30. Torgerson J, Hauptman J, Boldrin M, Sjstrm L. Xenical in the prevention of diabetes in obese subjects (XENDOS) study: a randomized study of orlistat as an adjunct to lifestyle changes for the prevention of type 2 diabetes in obese patients. Diabetes Care 2004;27:155-61.

31. Slanc P, Doljak B, Kreft S, Lunder M, Janes D, Strukelj B. Screening of selected food and medicinal plant extracts for pancreatic lipase inhibition. Phytother Res 2009;23:874-7.

32. Slanc P, Doljak B, Mlinaric A, Strukelj B. Screening of wooddamaging fungi and macrofungi for inhibitors of pancreatic lipase. Phytother Res 2004;18:758-62.

33. Bitou N, Ninomiya M, Tsujita T, Okuda H. Screening of lipase inhibitors from marine algae. Lipids 1999;34:441-5.

34. Keith SW, Redden DT, Katzmarzyk PT, Boggiano MM, Hanlon EC, Benca RM, et al. Putative contributors to the secular increase in obesity: exploring the roads less travelled. Int J Obes 2006;30:1585-94.

35. Singh S, Jarial R, Kanwar SS. Therapeutic effect of herbal medicines on obesity: herbal pancreatic lipase inhibitors. Wudpecker J Med Plants 2013;2:53-65.

36. Pollutants linked to reduced height and body mass; 2011. Available from: http://ec.europa.eu.environment/integration/ research/news alert/pdf/232na4. pdf]. [Last accessed on 02 Aug 2016].

37. Verhulst SL, Nelen V, Hond ED, Koppen G, Beunckens C, Vael C, Schoeters G, et al. Intrauterine exposure to environmental pollutants and body mass index during the first $3 \mathrm{Y}$ of life. Environ Health Perspect 2009;117:122-6. 
38. Herman CP. Effects of heat on appetite, National Academy Press: Washington DC; 1993.

39. Westerterp-Plantenga MS, Marken-Lichtenbelt WD, Cilissen C, Top S. Energy metabolism in women during short exposure to the thermoneutral zone. Physiol Behav 2002;75:227-35.

40. Flegal KM, TroianoRP, Pamuk ER, Kuczmarski RJ, Campbell SM. The influence of smoking cessation on the prevalence of overweight in the United States. N Engl JMed 1995;333:1165-70.

41. McGovern JA, Benowitz NL. Cigarette smoking, nicotine and body weight. Clin Pharmacol Ther 2011;90:164-8.

42. European commission DG ENV. Pollutants linked to reduced height and body mass; 2011. Available from: http://ec.europa.eu.environment/integration/research/newsa lert/pdf/232na4.pdf. [Last accessed on 02 Aug 2016]

43. Ravussin E, Valencia ME, Esparza J, Bennett PH, Schultz LO. Effects of a traditional lifestyle on obesity in Pima Indians. Diabetes Care 1994;17:1067-74.

44. Kawate R, Yamakido M. Nishimoto migrant studies among the Japanese in Hiroshima and Hawaii. Proc 10th Congr Int Diabetes Federation; 1980. p. 526-31.

45. Von Kries R, Toschke AM, Wurmser H, Sauerwald T, Koletzko B. Reduced risk for overweight and obesity in 5-and 6-year old children by duration of sleep: a cross-sectional study. Int J Obesity Related Metab Disorders 2002;26:710-6.

46. Taheri S, Lin L, Austin D, Young T, Mignot E. Short sleep duration is associated with reduced leptin, elevated ghreln, and increased body mass index. PLoS Med Epub 2004;1:210-7.

47. Gangwisch JE, Malaspina D, Boden-Albala B, Heymsfield SB. Inadequate sleep as a risk factor for obesity. Anal NHANES 2005;28:1289-96.

48. O'Rahilly S, Farooqi IS. Genetics of obesity. Philos Trans R Soc Lond B Biol Sci 2006;361:1095-105.

49. Loos RJ, Bouchard C. FTO: the first gene contributing to common forms of human obesity. Obes Rev 2008;9:246-50.

50. Farooqi IS, Keogh JM, Yeo GSH, Lank EJ, Cheetham T, O’Rahilly S. Clinical spectrum of obesity and mutations in the melanocortin 4 receptor gene. Engl J Med 2003;348:1085-95.

51. Nothen MM, Cichon S, Hemmer S, Hebebrand J, Remschmidt H, Lehmkuhl G, et al. Human dopamine D4 receptor gene: frequent occurrence of a null allele and observation of homozygosity. Hum Mol Genet 1994;3:2207-12.

52. Ristow M, Muller-Wieland D, Pfeiffer A, Krone W, Kahn CR. Obesity associated with a mutation in a genetic regulator of adipocyte differentiation. N Engl J Med 1998;339:953-9.

53. Campfield LA, Smith FJ, Rosenbaum M, Hirsch J. Human eating: evidence for a physiological basis using a modified paradigm. Neurosci Biobehav Rev 1996;20:133-7.

54. Bouchard C. The biological predisposition to obesity: beyond the thrifty genotype scenario. Int J Obes 2007;31:1337-9.

55. Jensen TK, Andersson AM, Jorgensen N, Andersen AG, Carlsen $\mathrm{E}$, Petersen $\mathrm{JH}$, et al. Body mass index in relation to semen quality and reproductive hormones among 1,558 Danish men. Fertil Steril 2004;82:863-70.

56. Diaz J, Taylor EM. Abnormally high nourishment during sensitive periods results in body weight changes across generations. Obes Res 1998;6:368-74.

57. Weng HH, Bastian LA, Taylor DH, Moser BK, Ostbye T. Number of children associated with obesity in middle-aged women and men: results from the health and retirement study. J Women's Health 2004;13:85-91.

58. Bujjirao IG, Ratna Kumar PL. Anti-obese therapeutics from medicinal plants a review. Int J Bioassays 2013;2:1399-406.

59. George P, Nimmi OS. Cent percent safe centum plants for antiobesity. Int J Innovative Technol Creative Engineering 2011;1:1-19.

60. Strychar I. Diet in the management of weight loss. CMAJ 2006;174:56-62.

61. Ness-Abramof Rl, Apovian CM. Diet modification for treatment and prevention of obesity. Endocrine 2006;29:5-9.

62. Salmon J, Timperio A. Prevalence, trends and environmental influences on child and youth physical activity. Med Sports Sci 2007;50:183-99.

63. Rose'n T, Bosaeus I, To'lli J, Lindstedt G, Bengtsson BA. Increased body fat mass and decreased extracellular fluid volume in adults with growth hormone deficiency. Clin Endocrinol 1993;38:63-71.

64. Haslam DW, James WP. Obesity. Lancet 2005;366:1197-209.

65. Chiles C, Van Wattum PJ. Psychiatric aspects of the obesity crisis. Psychiatr Times 2010;27:47-51.

66. Brown CD, Higgins M, Donato KA, Rohde FC, Garrison R, Obarzanek E, et al. Body mass index and the prevalence of hypertension and dyslipidemia. Obes Res 2000;8:605-19.

67. Reisin E, Frohlich ED, Messerli FH, Dreslinski GR, Dunn FG, Jones $\mathrm{MM}$, et al. Cardiovascular changes after weight reduction in obesity hypertension. Ann Intern Med 1983;98:315-9.

68. Tuck MI, Sowers J, Dornfield L, Kledzik G, Maxwell M. The effect of weight reduction on blood pressure plasma renin activity and plasma aldosterone level in obese patients. N Engl J Med 1981;304:930-3.

69. Rocchini AP, Key J, Bondie D, Chico R, Moorehead C, Katch V, et al. The effect of weight loss on the sensitivity of blood pressure to sodium in obese adolescents. N Engl J Med 1989;321:580-5.

70. Landsberg L, Krieger DR. Obesity, metabolism and the sympathetic nervous system. Am J Hypertension 1989;2:125-32.

71. Jacobs DB, Sowers JR, Hmeidan A, Niyogi T, Simpson L, Standley PR. Effects of weight reduction on cellular cation metabolism and vascular resistance. Hypertension 1993;21:308-14.

72. Frohlich ED, Apstein C, Chobanian AV, Devereux RB, Dustan HP, Dzau V, et al. The heart in hypertension. N Engl J Med 1992;327:998-1008.

73. Stumvoll M, Goldstein BJ, Van Haeften TW. Type 2 diabetes: pathogenesis and treatment. Lancet 2008;371:2153-6.

74. Boden G, Lebed B, Schatz M, Homko C, Lemieux S. Effects of acute changes of plasma free fatty acids on intramyocellular fat content and insulin resistance in healthy subjects. Diabetes 2001;50:1612-7.

75. Marchesini G, Brizi M, Bianchi G, Tomassetti S, Bugianesi E. Nonalcoholic fatty liver disease: a feature of the metabolic syndrome. Diabetes 2001;50:1844-50.

76. Ravussin E, Smith SR. Increased fat intake, impaired fat oxidation, and failure of fat cell proliferation result in ectopic fat storage, insulin resistance, and type 2 diabetes mellitus. Ann N Y Acad Sci 2002;967:363-78.

77. Haffner SM, Mitchell BD, HazudaHP, Stern MP. Greater influence of the central distribution of adipose tissue on the incidence of non-insulin-dependent diabetes in women than men. Am J Clin Nutr 1991;53:1312-7.

78. Health implications of obesity. National institutes of health consensus development conference statement. Ann Intern Med 1985;103:1073-7.

79. Hubert HB, Feinleib M, McNamara PM, Castelli WP. Obesity as an independent risk factor for cardiovascular disease: a 26year follow-up of participants in the Framingham Heart Study. Circulation 1983;67:968-77.

80. Lavie CJ, Milani RV, Ventura HO. Obesity and cardiovascular disease: risk factor, paradox, and impact of weight loss. J Am Coll Cardiol 2009;53:1925-32.

81. Messerli FH, Nunez BD, Ventura HO, Snyder DW. Overweight and sudden death. Increased ventricular ectopy in cardiopathy of obesity. Arch Intern Med 1987;147:1725-28.

82. Lavie CJ, McAuley PA, Church TS, MD Milani RV, Blair SN. Obesity and cardiovascular diseases implications regarding fitness, fatness, and severity in the obesity paradox. J Am College Cardiol 2014;63:1345-54.

83. Poulain M, Doucet M, Major GC, Drapeau V, Se'rie`s F. The effect of obesity on chronic respiratory diseases: pathophysiology and therapeutic strategies. CMAJ 2006;174:1293-9.

84. Donohoe CL, Pidgeon GP, Lysaght J, Reynolds JV. Obesity and gastrointestinal cancer. Br J Surg 2010;97:628-42.

85. Bao B, Wang Z, Li Y, Kong D, Ali S, Banerjee S, et al. The complexities of obesity and diabetes with the development and progression of pancreatic cancer. Biochim Biophys Acta 2011;1815:135-46.

86. $\mathrm{Xu} \mathrm{Q}$ Anderson $\mathrm{D}$, Lurie-Beck J. The relationship between abdominal obesity and depression in the general population: a systematic review and meta-analysis. Obes Res Clin Pract 2011;5:267-78.

87. Mazza M, Marano G, Traversi G, Bria P, Mazza S. Primary cerebral blood flow deficiency and Alzheimer's disease: shadows and lights. J Alzheimer Dis 2011;23:375-89. 
88. Thompson WG, Cook DA, Clark MM, Levine JA. Concise review for clinicians. Mayo Clin Proc 2007;82:93-103.

89. Sahib NG, Saari N, Ismail A, Khatib A, Mahomoodally F, Abdul A. Plants'metabolites as potential anti-obesity agents. Sci World J 2012;1-8. Doi: 10.1100/2012/436039

90. Barsh GS, Farooqi IS, O'Rahilly S. Genetics of body weight regulation. Nature 2000;404:644-51.

91. Tziomalos K, Krassas GE, Tzotzas T. The use of sibutramine in the management of obesity and related disorders: an update. Vasc Health Risk Manag 2009;5:441-52.

92. Heal DJ, Aspley S, Prow MR, Jackson HC, Martin KF, Cheetham SC. Sibutramine: a novel anti-obesity drug. A review of the pharmacological evidence to differentiate it from damphetamine and d-fenfluramine. Int J Obes Relat Metab Disord 1998;22:28-9.

93. Liu YL, Heal DJ, Stock MJ. Mechanism of the thermogenic effect of Metabolite 2(BTS 54 505), a major pharmacologically active metabolite of the novel anti-obesity drug, sibutramine. Int J Obes Relat Metab Disord 2002;26:1245-53.

94. Nisoli E, Carruba MO. An assessment of the safety and efficacy of sibutramine, an anti-obesity drug with a novel mechanism of action. Obes Rev 2000;1:127-39.

95. Arterburn DE, Crane PK, Veenstra DL. The efficacy and safety of sibutramine for weight loss: a systematic review. Arch Int Med 2004;164:994-1003.

96. Fujioka K, Seaton TB, Rowe E, Jelinek CA, Raskin P, Lebovitz HE, et al. Weight loss with sibutramine improves glycaemic control and other metabolic parameters in obese patients with type 2 diabetes mellitus. Diabetes Obes Metab 2000;2:175-87.

97. Meridia (sibutramine hydrochloride). American Society of Health-System Pharmacists; 2012. http://fda.gov/Drugs/ DrugSafety/PostmarketDrugSafetyInformationforPatientsandP roviders/ucm191652.htm. [Last accessed on 03 Aug 2016]

98. Ballinger A, Peikin SR. Orlistat: its current status as an antiobesity drug. Eur J Pharmacol 2002;440:109-17.

99. Neovius M, Johans SK, Rssner S. Head-to-head studies evaluating the efficacy of pharmacotherapy for obesity: a systematic review and meta-analysis. Obes Rev 2008;9:420-7.

100. Torgerson JS. Xenical in the prevention of edicao especialoutubro 2010-ABESO 47-17 diabetes in obese subjects (XENDOS) study: a randomised study of orlistat as an adjunct to lifestyle changes for the prevention of type 2 diabetes in obese patients. Diabetes Care 2004;27:155-61.

101. Van-Gaal L. "Efficacy and tolerability of orlistat in the treatment of obesity: a 6-month dose-ranging study. Orlistat Dose-Raging Study Group". Eur J Clin Pharmacol 1998;54:125-32.

102. Kaila B, Raman M. Obesity: a review of pathogenesis and management strategies. Can J Gastroenterol 2008;22:61-8.

103. Lam DD, Przydzial MJ, Ridley SH, Yeo GS, Rochford JJ, O’Rahilly $S$, et al. Serotonin 5-HT2C receptor agonist promotes hypophagia via downstream activation of melanocortin 4 receptors. Endocrinology 2008;149:1323-8.

104. Bray GA, Greenway FL. Current and potential drugs for the treatment of obesity. Endocr Rev 1999;20:805-75.

105. Croft H, Houser TL, Jamerson BD, Leadbetter R, Bolden-Watson C, Donahue R, et al. Effect on body weight of bupropin sustained-release in patients with major depression treated for 52 w. Clin Ther 2002;24:662-72.

106. Scheen AJ, Paquot N. Use of cannabinoid CB1 receptor antagonists for the treatment of metabolic discords. Best Pract Res Clin Endocrinol Metab 2009;23:103-6.

107. Mechanick JI. Metabolic complications of baritic surgery. Proceedings: of the 16th Annual Meeting of the American Association of Clinical Endocrinologists (AACE) Seattle; 2007. p. 89-94.

108. Maciejewski ML, Livingston EH, Smith VA, Kavee AL, Kahwati LC, Henderson WG, Arterburn DE. Survival among high-risk patients after bariatric surgery. JAMA 2011;305:2419-26.

109. Singh S, Jarial R, Kanwar SS. Therapeutic effect of herbal medicines on obesity: herbal pancreatic lipase inhibitors. Wudpecker J Med Plants 2013;2:53-65.

110. Park MY, Lee KS, Sung MK. Effects of dietary mulberry, Korean red ginseng, and banaba on glucose homeostasis in relation to PPAR-a, PPAR-c, and LPL mRNA expressions. Life Sci 2005;77:3344-54.
111. Nakayama T, Suzuki S, Kudo H, Sassa S, Nomura M, Sakamoto S. Effects of three Chinese herbal medicines on plasma and liver lipids in mice fed a high fat diet. J Ethnopharmacol 2007;109:236-40.

112. Rayalam S, Della-Fera MA, Baile CA. Phytochemicals and regulation of the adipocyte life cycle. J Nutr Biochem 2008;19:717-26.

113. Williams DJ, Edwards D, HamernigI, Jian I, James AP, Johnson SK, TapsellLC. Vegetables containing phytochemicals with potential anti-obesity properties: a review. Food Res Int 2013;52:323-33.

114. Thielecke F, Boschmann M. The potential role of green tea catechins in the prevention of the metabolic syndrome-a review. Phytochemistry 2009;70:11-24.

115. Wolfram S, Wang Y, Thielecke F. Anti-obesity effects of green tea: from bedside to bench. Mol Nutr Food Res 2006;50:17687.

116. Birari RB, Bhutani KK. Pancreatic lipase inhibitors from natural sources: unexplored potential. Drug Discovery Today 2007;12:879-89.

117. Sukhdev S, Singh KS. Therapeutic role of phytomedicines on obesity: the importance of herbal pancreatic lipase inhibitors. Int Res J Med Sci 2013;1:15-26.

118. Hadvary P, Lengsfeld $H$, WolferH. Inhibition of pancreatic lipase in vitro by the covalent inhibitor tetrahydrolipstatin. Biochem J 1998;256:357-61.

119. Hadvary P, Sidler W, Meister W, Vetter, Wolfer H. The lipase inhibitor tetrahydrolipstatin binds covalently to the putative active site serine of pancreatic lipase. J Biol Chem 1991;266:2021-7.

120. Tsujita T, Takaichi H, Takaku T, Aoyama S, Hiraki J. Anti-obesity action of e-polylysine, a potent inhibitor of pancreatic lipase. J Lipid Res 2006;47:1852-8.

121. Karamadoukis L, Shivashankar GH, Ludeman L, Williams AJ. An unusual complication of treatment with orlistat. Clin Nephrol 2009;71:430-2.

122. Thurairajah PH, Syn WK, Neil DA, Stell D, Haydon G. Orlistat (xenical)-induced subacute liver failure. Eur J Gastroenterol Hepatol 2005;17:1437-8.

123. Chaput JP, St-Pierre S, Tremblay A. Currently available drugs for the treatment of obesity: sibutramine and orlistat. Mini Rev Med Chem 2007;7:3-10.

124. Yuniarto A, Kurnia I, Ramadhan M. Anti-obesity effect of ethanolic extract of jasmine flowers (Jasminumsambac L.) in high-fat diet induced mice: a potent inhibitor of pancreatic lipase enzyme. Int J Adv Pharm Biol Chem 2015;4:18-22.

125. Ong SL, Paneerchelvan S, Lai HY, Rao NK. In vitro lipase inhibitory effect of thirty-two selected plants in Malaysia. Asian J Pharm Clin Res 2014;7:19-24.

126. Adnyana IK, Sukandar EY, Yuniarto A, Finna S. Anti-obesity effect of the pomegranate leaves ethanol extract (punicagranatum L.) In high-fat diet induced mice. Int J Pharm Pharm Sci 2014;6:626-31.

127. Bujjirao G, Ratnakumar PK, Shaik KB. Evaluation of anti-obesity properties of few south indian medicinal plants by using pancreatic lipase assay. Int J Chem Life Sci 2014;22:34-8.

128. Changhyun R, Uhee J. Screening of crude plant extracts with anti-obesity activity. Int J Mol Sci 2012;13:1710-9.

129. Lee YM, Kim YS, Lee YS, Kim J, Sun HJ, Kim JH, et al. Inhibitory activities of pancreatic lipase and phosphodiesterase from korean medicinal plant extracts. Phytother Res Phytother Res 2012;26:778-82.

130. Bustanji Y, Mohammad M, Hudaib M, Tawaha K, Al-Masri IM, Alkhatib HS, et al. Screening of some medicinal plants for their pancreatic lipase inhibitory potential. Jordan J Pharm Sci 2011;4:81-8.

131. Zheng CD, Duan YQ, Gao JM, Ruan ZG. Screening for anti-lipase properties of 37 traditional chinese medicinal herbs. J Chin Med Assoc 2010;73:319-24.

132. Han LK, Zheng YN, Yoshikawa M, Okuda H, Kimura Y. Anti-obesity effects of chikusetsusaponins isolated from Panax japonicus rhizomes. BMC Complementary Altern Med 2005;5:1472-82.

133. Han LK, Xu BJ, Kimura Y, Zheng Y, Okuda H. Platycodi radix affects lipids metabolism in mice with fat diet-induced obesity. J Nutr 2000;130:2760-4. 
134. Kishino E, Ito T, Fujita K, Kiuchi Y. A mixture of the Salacia reticulate (kotala himbutu) aqueous extract, and cyclodextrin reduces the accumulation of visceral fat mass in mice and rats with high-fat-diet-induced obesity. J Nutr 2006;136:433-9.

135. Ono Y, Hattori E, FukayaY, Imai S, Ohizumi Y. Anti-obesity effect of Nelumbo nucifera leaves extract in mice and rats. J Ethnopharmacol 2006;106:238-44.

136. Kim HY, Kang MH. Screening of Korean medicinal plants for lipase inhibitory activity. Phytother Res 2005;19:359-61.

137. Han LK, Nose R, Li W, Gong XJ, Zhen YN, Yoshikawa M, et al. Reduction of fat storage in mice fed a high-fat diet long term by treatment with saponin prepared from kochia scoparia fruit. Phytother Res 2006;20:877-82.

138. Moreno DA, IIic N, Pouley A, Raskin I. Effects of Arachis hypogaea nutshell extract on lipid metabolic enzymes and obesity parameters. Life Sci 2006;78:2797-803.

139. Shimoda H, Seki E, Aitani M. Inhibitory effect of green coffee bean extract on fat accumulation and body weight gain in mice. BMC Complementary Altern Med 2006;6:1-9.

140. Takao I, Fujii S, Ishii A, Han LK, Kumao T, Ozaki K. Effects of mannooligosaccharides from coffee mannan on fat storage in mice fed a high-fat diet. J Health Sci 2006;52:333-7.

141. Han LK, Kimura Y, Okuda H. Anti-obesity effects of natural products. Stud Nat Prod Chem 2005;30:79-110.

142. Bonodiolotti G, Bareggi SR, Frega NG, Strabioli S, Cornelli U. Activity of two diffent poly glucosamines, L112 and FF45, on body weight in male rats. Eur J Pharmacol 2007;567:155-8.

143. Gades MD, Stern JS. Chitosan supplementation and fecal fat excretion in men. Obes Res 2003;11:683-8.

144. Gades MD, Stern JS. Chitosan supplementation and fat absorption in men and women. J Am Diet Assoc 2005;105:72-7.

145. Gallaher DD, Gallaher CM, Mahrt GJ, Carr TP, Hollingshead CH, Hesslink R, et al. A glucomannan and chitosan fiber supplement decreases plasma cholesterol and increases cholesterol excretion in overweight normocholesterolemic humans. J Am Coll Nutr 2002;21:428-33.

146. Han LK, Kimura Y, Okuda H. Reduction in fat storage during chitin-chitosan treatment in mice fed a high-fat diet. Int J Obes Relat Metab Disord 1999;23:174-9.

147. Hayashi K, Ito M. Antidiabetic action of low molecular weight chitosan in genetically obese diabetic KK-Ay mice. Biol Pharm Bull 2002;25:188-92.

148. Ho SC, Tai ES, Eng PH, Tan CE, Fok AC. In the absence of dietary surveillance, chitosan does not reduce plasma lipids or obesity in hypercholesterolaemic obese Asian subjects. Singapore Med J 2001;42:6-10.

149. Kaats GR, Michalek JE, Preuss HG. Evaluating efficacy of a chitosan product using a double-blinded, placebo-controlled protocol. J Am Coll Nutr 2006;25:389-94.

150. Sumiyoshi M, Kimura Y. Low molecular weight chitosan inhibits obesity induced by feeding a high-fat diet long-term in mice. J Pharm Pharmacol 2006;58:201-7.

151. Weibel EK, Hadvary P, Hochuli E, Kupfer E, Lengsfeld H. Lipstatin, an inhibitor of pancreatic lipase, produced by Streptomyces toxytricini. I. Producing organism, fermentation, isolation and biological activity. J Antibiot (Tokyo) 1987;40:1081-5.

152. Mutoh M, Nakada N, Matsukuma S, Ohshima S, Yoshinari K, Watanabe J, et al. Panclicins, novel pancreatic lipase inhibitors. I. Taxonomy, fermentation, isolation and biological activity. J Antibiot (Tokyo) 1994;47:1369-75.

153. Kitahara M, Asano M, Naganawa H, Maeda K, Hamada M, Aoyagi $\mathrm{T}$, et al. Valilactone. An inhibitor of esterase produced by actinomycetes. J Actinomycetes J Antibiot (Tokyo) 1987; 40:1647-50.

154. Umezawa $H$, Aoyagi $T$, Uotani $K$, Hamada $M$, Takeuchi $T$, Takahashi S. Ebelactone, an inhibitor of esterase, produced by actinomycetes. J Antibiot (Tokyo) 1980;33:1594-6.

155. Umezawa H, Aoyagi T, Hazato T, Uotani K, Kojima F, Hamada M, et al. An inhibitor of esterase, produced by actinomycetes. J Antibiot (Tokyo) 1978;31:639-41.

156. Tomoda H, Namatame I, Omura S. Microbial metabolites with inhibitory activity against lipid metabolism. Proc Japan Acad 2002;78:217-40.
157. Liu DZ, Wang F, Liao TG, Tang JG, Steglich W, Zhu HJ, et al Vibralactone: a lipase inhibitor with an unusal fused bêtelactone produced by cultures of the basidiomycete Boreostereum vibrans. Org Lett 2006;8:5749-52.

158. Bitou N, Ninomiya M, Tsujita T, Okuda H. Screening of lipase inhibitors from marine algae. Lipids 1999;34:441-5.

159. Ahn MY, Jee SD, Lee BM. Anti-obesity effects of Isaria sinclairii by repeated oral treatment in obese zucker rats over a 4-mouth period. J Toxicol Environ Health 2007;70:1395-401.

160. Slanc P, Doljak B, Mlinaric A, Strukelj B. Screening of wooddamaging fungi and macrofungi for inhibitors of pancreatic lipase. Phytother Res 2004;18:758-62.

161. Lin JK, Lin-Shiau SY. Mechanisms of hypolipidemic and antiobesity effects of tea and tea polyphenols. Mol Nutr Food Res 2006;50:211-7.

162. Nakai M, Fukui Y, Asami S, Toyoda-Ono Y, Iwashita T, Shibata H, et al. Inhibitory effects of oolong tea polyphenols on pancreatic lipase in vitro. J Agric Food Chem 2005;53:4593-8.

163. Han LK, Takaku T, Li J, Kimura Y, Okuda H. Anti-obesity action of oolong tea. Int J Obesity Related Metab Disorders 1999;23:98-105.

164. Zhao HL, Sim JS, Shim SH, Ha YW, Kang SS, Kim YS. Antiobese and hypolipidemic effects of platycodin saponins in dietinduced obese rats: evidence for lipase inhibition and calorie intake restriction. Int J Obes 2005;29:983-90.

165. Zhao HL, Kim YS. Determination of the kinetic properties of platycodin for the inhibition of pancreatic lipase using a 1,2diglyceride-based colorimetric assay. Arch Pharm Res 2004;27:968-72.

166. Han LK, Zheng YN, XuBj, Okuda H, Kimura Y. Saponins from platycodi radix ameliorate high fat diet-induced obesity in mice. J Nutr 2002;132:2241-5.

167. Xu BJ, Han LK, Zheng YN, Lee JH, Sung CK. In vitro inhibitory effect of triterpenoidal saponins from Platycodi radix on pancreatic lipase. Arch Pharm Res 2005;28:180-5.

168. Lei F, Zhang XN, Wang W, Xing DM, Xie WD, Su H, et al. Evidence of anti-obesity effects of the pomegranate leaf extract in high-fat diet induced obese mice. Int J Obes 2007;31:1023-9.

169. Yamamoto $M$, Shimura $S$, Itoh $Y$, Ohsaka T, Egawa M, Inoue S. Anti-obesity effects of lipase inhibitor CT-II, an extract from edible herbs, nomame herba, on rats fed a high-fat diet. Int J Obesity Related Metab Disorders 2000;24:758-64.

170. Ekanem AP, Wang M, Simon JE, Moreno DA. Antiobesity properties of two African plants (Afromomum meleguetta and Spilanthes acmella) by pancreatic lipase inhibition. Phytother Res 2007;21:1253-5.

171. Han LK, Kimura Y, Kawashima M, Takaku T, Taniyama T, Hayashi T, et al. Anti-obesity effects in rodents of dietary teasaponin, a lipase inhibitor. Int J Obes Relat Metab Disord 2001;25:1459-64.

172. Handa T, Sono YY, Yazawa K. Effects of fenugreek seed extract in obese mice fed a high-fat diet. Biosci Biotechnol Biochem 2005;69:1186-8.

173. Han LK, Sumiyoshi M, Zhang J, Liu MX, Zhang YN, Okuda H, et al. Anti-obesity action by polyphenols of Salix matsudana in highfat-diet treated rodent animals. Phytother Res 2003;17:1188-94.

174. Han LK, Sumiyoshi M, Zhang YN, Okuda H, Kimura Y. Isolation of anti-obesity effectors from polyphenol fraction of Salix matsudana. Phytother Res 2003;17:1195-8.

175. Sharma N, Sharma VK, Seo SY. Screening of some medicinal plants for anti-lipase activity. J Ethnopharmacol 2005;97:453-6.

176. Ninomiya K, Matsuda $H$, Shimoda H, Nishida N, Kasajima N, Yoshino T, et al. Carnosic acid, a new class of lipid absorption inhibitor from sage. Bioorg Med Chem Lett 2004;14:1943-6.

177. Zheng Q, Koike K, Han LK, Okuda H, Nikaido T. New biologically active triterpenoid saponins from Scabiosa tschiliensis. J Nat Prod 2004;67:604-13.

178. Yoshizumi K, Hirano K, Ando H, Hirai Y, Ida Y, Tsuji T, et al. Lupane-type saponins from leaves of Acanthopanax sessiliflorus and their inhibitory activity on pancreatic lipase. J Agric Food Chem 2006;54:335-41.

179. Kimura H, Ogawa S, Jisaka M, Kimura Y, Katsube T, Yokota K. Identification of novel saponins from edible seeds of Japanese horse chestnut (Aesculus turbinate Blume) after treatment 
with wooden ashes and their nutraceutical activity. J Pharm Biomed Anal 2006;41:1657-65.

180. Kurihara H, Asami S, Shibata H, Fukami H, Tanaka T. Hypolipemic effect of Cyclocarya paliurus (batal) iljinskaja in lipid-loaded mice. Biol Pharm Bull 2003;26:383-5.

181. Deshpande MS, Shengule S, Apte KG, Wani M, Piprode V, Parab PB. Anti-Obesity Activity Of Ziziphus Mauritiana: A Potent Pancreatic Lipase Inhibitor. Asian J Pharm Clin Res 2003;6:168-73.

182. Lee IA, Lee JH, Baek NI, Kim DH. Antihyperlipidemic effect of crocin isolated from the fructus of Gardenia jasminoides and its metabolite Crocetin. Biol Pharm Bull 2005;28:2106-10.

183. Sheng L, Qian Z, Zheng S, Xi L. Mechanism of hypolipidemic effect of crocin in rats: crocin inhibits pancreatic lipase. Eur J Pharmacol 2006;543:116-22.

184. Kwon CS, Sohn HY, Kim SH, Kim JH, Son KH, Lee JS, et al. Antiobesity effect of Dioscorea nipponica makino with lipase inhibitory activity in rodents. Biosci Biotechnol Biochem 2003;67:1451-6.

185. Won SR, Kim SK, Kim YM, Lee PH, Ryu JH, Kim JW, et al. Licochalcone A: lipase inhibitor from the roots of Glycyrrhiza uralensis. Food Res Int 2007;40:1046-50.

186. Kannan M, Dheeba B, Nageshwari K, Kannan K, Venkatesan S. Antibacterial and anti-obesity activities of marine algae Gracilaria corticata and Spirulina platensis. Int J Pharm Pharm Sci 2014;6:420-4.

187. Slanc P, Doljak B, Mlinaric A, Strukelj B. Screening of wooddamaging fungi and macrofungi for inhibitors of pancreatic lipase. Phytother Res 2004;18:758-62.

188. Kawaguchi K, Mizuno T, Aida K, Uchino K. Hesperidin as an inhibitor of lipase from porcine pancreas and pseudomonas. Biosci Biotechnol Biochem 1997;61:102-4.

189. Lean ME. How does sibutramine work? Int J Obes Relat Metab Disord 2001;4:8-11

190. Poston WS, Foreyt JP. Sibutramine and the management of obesity. Expert Opin Pharmacother 2004;5:633-42.

191. Chantre P, Lairon D. Recent findings of green tea extract AR25 (exolise) and its activity for the treatment of obesity. Phytomedicine 2002;9:3-8.

192. Halford JC, Blundell JE. Pharmacology of appetite suppression. Prog Drug Res 2000;54:25-58.

193. Wynne K, Stanley S, McGowan BB. Appetite control. J Endocrinol 2005;184:291-318.

194. Van Heerden FR. Hoodia gordonii: a natural appetite suppressant. J Ethnopharmacol 2008;119:434-7.

195. Ohia SE, Opere CA, Leday AM, Bagch M, Bagchi D, Stohs SJ. Safety and mechanism of appetite suppression by a novel hydroxy citric acid extract (HCA-SX). Mol Cell Biochem 2002;238:89-103.

196. Lane D, Cha SH. Effect of glucose and fructose on food intake via malonyl-CoA signalling in the Brain. Biochem Biophys Res Commun 2009;382:1-5.

197. Cha SH, Wolfgang M, Tokutake Y, Chohnan S, Lane MD. Differential effects of central fructose and glucose on hypothalamic malonyl-CoA and food intake. Proc Natl Acad Sci USA 2008;105:16871-5.

198. Ambadasu B, Dange SV, Wali RS. Effect of Caralluma fimbriata extract on appetite, body weight and lipid profile in cafeteria dietinduced obesity in rats. Int J Pharm Pharm Sci 2013;5:536-9.

199. Kim JH, Hahm DH, Yang DC, Kim JH, Lee HJ, Shim I. Effect of crude saponin of Korean red ginseng on high-fat-diet-induced obesity in the rat. J Pharmacol Sci 2005;97:124-31.

200. Mopuri R, Ganjayi M, Banavathy KS, Parim BN, Meriga B. Evaluation of anti-obesity activities of ethanolic extract of Terminalia paniculata bark on high fat diet-induced obese rats. BMC Complementary Altern Med 2015;15:2-11.

201. Prashar Y, Kumar AS. Anti-obesity activity of Bauhinia variegata Linn. in high-fat diet-induced obesity in female rats. Pharmacologyonline 2010;2:1008-16.

202. Kim YJ, Shin YO, Ha YW, Lee S, Oh JK, Kim YS. Anti-obesity effect of Pinellia ternata extract in zucker rats. Biol Pharm Bull 2006;29:1278-81.

203. Saito M, Ueno M, Ogino S, Kubo K, Nagata J, Takeuchi M. High dose of Garcinia cambogia is effective in suppressing fat accumulation in developing male Zucker obese rats, but highly toxic to the testis. Food Chem Toxicol 2005;43:411-9.

204. Moon HS, Lee HG, Choi YJ, Kim TG, Cho CS. Proposed mechanisms of (_) epigallocatechin-3-gallate for anti-obesity. Chem Biol Interact 2007;167:85-98.

205. MacLean DB, Luo LG. Increased ATP content/production in the hypothalamus may be a signal for energy-sensing of satiety: studies of the anorectic mechanism of a plant steroidal glycoside. Brain Res 2004;1020:1-11.

206. Pereira CA, Pereira LLS, Correa AD. Hoodia gordonii in the treatment of obesity: a review. J Med Plants Res 2010;4:2305-12.

207. Baintner K, Kiss P, Pfu“ ller U, Bardocz S, Pusztai A. Effect of orally and intraperitoneally administered plant lectins on food consumption of rats. Acta Physiol Hung 2003;90:97-107.

208. Hughes GM, Boyland EJ, Williams NJ, Mennen L, Scott C, Kirkham TC, et al. The effect of Korean pine nut oil (PinnoThin) on food intake, feeding behaviour and appetite: a double-blind placebo-controlled trial. Lipids Health Dis 2008;7:1-10.

209. Fleming RM. The effect of ephedra and high-fat dieting: a cause for concern! A case report. Angiology 2007;58:102-5.

210. Klontz KC, Timbo BB, Street D. Consumption of dietary supplements containing Citrus aurantium (bitter orange)-2004 california behavioral risk factor surveillance survey (BRFSS). Ann Pharmacother 2006;40:1747-51.

211. Husain GM, Chatterjee SS, Singh PN, Kumar V. Hypolipidemic and anti-obesity-like activity of standardised extract of Hypericum perforatum L. in rats. Int Scholarly Res Network Pharmacol 2011;1-7. http://dx.doi.org/10.5402/2011/505247

212. Ohkoshi E, Miyazaki H, Shindo K, Watanabe H, Yoshida A Yajima H. Constituents from the leaves of Nelumbo nucifera stimulate lipolysis in the white adipose tissue of mice. Planta Med 2007;73:1255-9

213. Attele AS, Zhou YP, Xie JT, Wu JA, Zhang L, Pugh W, et al. Antidiabetic effects of panax ginseng berry extract and the of an effective component. Diabetes 2002;51:1851-8.

214. Moriyama T, Kishimoto K, Nagai K, Urade R, Ogawa T, Utsumi S, et al. Soybean $\beta$-conglycinin diet suppresses serum triglyceride levels in normal and genetically obese mice by induction of $\beta$-oxidation, downregulation of fatty acid synthase, and inhibition of triglyceride absorption. Biosci Biotechnol Biochem 2004;68:352-9.

215. Ishihar K, Oyaizu S, Fukuchi Y, Mizunoya W, Segawa K, Takahashi $M$, et al. A soybean peptide isolate diet promotes postprandial carbohydrate oxidation and energy expenditure in type-II diabetic mice. J Nutr 2003;133:752-7.

216. Maeda H, Hosokawa M, Sashima T, Funayama K, Miyashita K Fucoxanthin from edible seaweed, Undaria pinnatifida, shows anti-obesity effects through UCP1 expression in white adipose tissues. Bioche Biophys Res Commun 2005;332:392-7.

217. Maeda H, Hosokawa M, Sashima T, Funayama K, Miyashita K. Effect of medium-chain triacylglycerols on anti-obesity effects of fucoxanthin. J Oleo 2007;56:615-21.

218. Maeda H, Hosokawa M, Sashima T, Miyashita K. Dietary combination of fucoxanthin and fish oil attenuates the weight gain of white adipose tissue and decreases blood glucose in obese/diabetic KK-Ay mice. J Agric Food Chem 2007;55:7701-6.

219. Tsuboyama-Kasaoka N, Takahashi M, Kim H, Ezaki O. Upregulation of liver uncoupling protein-2 mRNA by either fish oil feeding or fibrate administration in mice. Biochem Biophys Res Commun 1999;257:879-85.

220. Ortinau LC, Nickelson KJ, Stromsdorfer KL, Naik CY, Pickering RT, Haynes RA, et al. Sterculic oil, a natural inhibitor of scd1, improves the metabolic state of obese oletf rats. Obesity 2013;21:344-52.

221. Flatt JP. Differences in basal energy expenditure and obesity. Obesity (Silver Spring) 2007;15:2546-8.

222. Redinger RN. Fat storage and the biology of energy expenditure. Transl Res 2009;154:52-60.

223. Cannon B, Nedergaad J. Brown adipose tissue: function and physiological significance. Physiol Rev 2004;84:277-359.

224. Kumar MV, Sunvold GD, Scarpace PJ. Dietary vitamin a supplementation in rats: suppression of leptin and induction of UCP1 mRNA. J Lipid Res 1999;40:824-9.

225. Yoon SS, Rhee YH, Lee HJ, Lee EO, Lee MH, Ahn KS, et al. Uncoupled protein 3 and p38 signal pathways are involved in 
the anti-obesity activity of Solanum tuberosum L. Cv. Bora Valley. J Ethnopharmacol 2008;118:396-404.

226. Kim HK, Della-Fera M, Lin J, Baile CA. Docosahexaenoic acid inhibits adipocyte differentiation and induces apoptosis in 3T3L1 preadipocytes. J Nutr 2006;136:2965-9.

227. Cowherd RM, Lyle RE, McGehee RE Jr. Molecular regulation of adipocyte differentiation. Semin Cell Dev Biol 1999;10:3-10.

228. Green $\mathrm{H}$, Kehinde 0 . An established a preadipose cell line and its differentiation in culture. II. Factors affecting the adipose conversion. Cell 1975;5:19-27.

229. Wu Z, Puigserver P, Spiegelman BM. Transcriptional activation of adipogenesis. Curr Opin Cell Biol 1999;11:689-94.

230. Lefterova MI, Lazarus MA. New developments in adipogenesis. Trends Endocrinol Metab 2009;20:107-14.

231. Awad AB, Begdache LA, Fink CS. Effect of sterols and fatty acids on growth and triglyceride accumulation in 3T3-L1 cells. J Nutr Biochem 2000;11:153-8.

232. Evans M, Geigerman C, Cook J, Kuebler B, McIntosh M. Conjugated linoleic acid suppresses triglyceride accumulation and induces apoptosis in 3T3-L1 preadipocytes. Lipids 2000;35:899-910.

233. Okuno M, Kajiwara K, Imai S, Kobayashi T, Honma N, Maki T, et al. Perilla oil prevents the excessive growth of visceral adipose tissue in rats by down-regulating adipocyte differentiation. J Nutr 1997;127:1752-7.

234. Madsen L, Petersen RK, Kristiansen K. Regulation of adipocyte differentiation and function by polyunsaturated fatty acids. Biochem Biophys Acta 2005;1740:266-86.

235. Shimada T, Hiramatsu N, Kasai A, Mukai M, Okamura M, Yao J, et al. Suppression of adipocyte differentiation by Cordyceps militaris through activation of the aryl hydrocarbon receptor. Am J Physiol Endocrinol Metab 2008;295:859-67.

236. Kim MS, Kim JK, Kwon DY, Park R. Anti-adipogenic effects of Garcinia extract on the lipid droplet accumulation and the expression of a transcription factor. Biofactors 2004;22:193-6.

237. Garg A, Singh R. Anti-obesity activity of ethanolic extract of Cassia auriculata in high-fat-diet-induced obese rats. Int J Pharm Pharm Sci 2015;7:237-43

238. Jeon JR, Kim JY. Effects of pine needle extract on the differentiation of 3T3-L1 preadipocytes and obesity in high-fat diet fed rats. Biol Pharm Bull 2006;29:2111-5.

239. Lee YS, Kim WS, Kim KH, Yoon MJ, Cho HJ, Shen Y, et al. Berberine, a natural plant product, activates AMP-activated protein kinase with beneficial metabolic effects in diabetic and insulin-resistant states. Diabetes 2006;55:2256-64.

240. Huang C, Zhang Y, Gong Z, Sheng X, Li Z, Zhang W, et al. Berberine inhibits 3T3-L1 adipocyte differentiation through the PPARgamma pathway. Biochem Biophys Res Commun 2006;348:571-8.

241. Hu Y, Davies GE. Berberine increases expression of GATA-2 and GATA-3 during inhibition of adipocytes differentiation. Phytomedicine 2009;16:864-73.

242. Hsu CL, Yen GC. Effects of capsaicin on induction of apoptosis and inhibition of adipogenesis in 3T3-L1 cells. J Agric Food Chem 2007;55:1730-6.

243. Liu F, Kim J, Li Y, Liu X, Li J, Chen X. An extract of Lagerstroemia speciosa L. has insulin-like glucose uptake-stimulatory and adipocyte differentiation-inhibitory activities in 3T3-L1 cells. J Nutr 2001;131:2242-7.

244. Liu X, Kim JK, Li Y, Li J, Liu F, Chen X. Tannic acid stimulates glucose transport and inhibits adipocyte differentiation in 3T3L1 cells. J Nutr 2005;135:165-71.

245. Bai N, He K, Roller M, Zheng B, Chen X, Shao Z, et al. Active compounds from Lagerstroemia speciosa, insulin-like glucose uptake stimulatory/inhibitory and adipocyte differentiationinhibitory activities in 3T3-L1 cells. J Agric Food Chem 2008;56:11668-74

246. Klein G, Kim J, Himmeldirk K, Cao Y, Chen X. Antidiabetes and anti-obesity activity of Lagerstroemia speciosa. Evid Based Complement Altern Med 2007;4:401-7.

247. Maeda H, Hosokawa M, Sashima T, Takahashi N, Kawada T, Miyashita K. Fucoxanthin and its metabolite, fucoxanthinol, suppress adipocyte differentiation in 3T3-L1 cells. Int Mol Med 2006;18:147-52.
248. Ku HC, Chang HH, Liu HC, Hsiao CH, Lee MJ, Hu YJ, et al. Green tea (-)-epigallocatechin gallate inhibits insulin stimulation of 3T3-L1 preadipocyte mitogenesis via the $67-\mathrm{kDa}$ laminin receptor pathway. Am J Physiol Cell Physiol 2009;297:121-32.

249. Lee MS, Kim CT, Kim IH, Kim Y. Inhibitory effects of green tea catechin on the lipid accumulation in 3T3-L1 adipocytes. Phytother Res 2008;23:1088-91.

250. Sakurai N, Mochizuki K, Kameji H, Shimada M, Goda T. (_) Epigallocatechin gallate enhances the expression of genes related to insulin sensitivity and adipocyte differentiation in 3T3-L1 adipocytes at an early stage of differentiation. Nutrition 2009;25:1047-56.

251. Lee YK, Lee WS, Hwang JT, Kwon DY, Surh YJ, Park OJ. Curcumin exerts antidifferentiation effect through AMPKa-PPAR-c in 3T3-L1 adipocytes and antiproliferative effect through AMPKa-COX-2 in cancer cells. J Agric Food Chem 2009;57:305-10.

252. Kubota H, Morii R, Kojima-Yuasa A, Huang X, Yano Y, MatsuiYuasa I. Effect of Zizyphus jujuba extract on the inhibition of adipogenesis in 3T3-L1 preadipocytes. Am J Chin Med 2009;37:597-608.

253. Ambati S, Yang JY, Rayalam S, Park HJ, Della-Fera MA, Baile CA. Ajoeneexerts potent effects in 3T3-L1 adipocytes by inhibiting adipogenesis and inducing apoptosis. Phytother Res 2009;23:513-8.

254. Yang JY, Della-Fera MA, Rayalam S, Baile CA. Effect of xanthohumol and isoxanthohumol on 3T3-L1 cell apoptosis and adipogenesis. Apoptosis 2007;12:1953-63.

255. Mendes V, Monterio R, Pestana D, Teixeira D, Calhau C, Azevado I. Xanthohumol influences preadipocyte differentiation: implication of antiproliferative and apoptotic effects. J Agric Food Chem 2008;56:11631-7.

256. Uto-Kondo H, Ohmori R, Kiyose C, Kishimoto Y, Saito H, Igarashi O, et al. Tocotrienol suppresses adipocyte differentiation and Akt phosphorylation in 3T3-L1 preadipocytes. J Nutr 2009;139:51-7.

257. Yamasaki M, Ogawa T, Wang L, Katsube T, Yamasaki Y, Sun X, et al. Anti-obesity effects of hot water extract from Wasabi (Wasabia japonica Matsum.) leaves in mice fed high-fat diets. Nutr Res Pract 2013;7:267-72.

258. Ito M, Ito J, Kitazawa H, Shimamura K, Fukami T, Tokita S, et al. Daisuke uemura (_) ternatin inhibits adipogenesis and lipid metabolism in 3T3-L1 cells. Peptides 2009;30:1074-81.

259. Xiong ZD, Li PG, Mu TH. The differentiation and proliferation inhibitory effects of sporamin from sweet potato in 3T3-L1 preadipocytes. Agric Sci Chin 2009;8:671-7.

260. Takahashi T, Tabuchi T, Tamaki Y, Kosaka K, Takikawa Y, Satoh T. Carnosic acid and carnosol inhibit adipocyte differentiation in mouse 3T3-L1 cells through induction of phase2 enzymes and activation of glutathione metabolism. Biochem Biophys Res Commun 2009;382:549-54.

261. Ejaz A, Wu D, Kwan P, Meydani M. Curcumin inhibits adipogenesis in 3T3-L1 adipocytes and angiogenesis and obesity in C57/BL mine. J Nutr 2009;139:919-25.

262. Miller M, Chen S, Woodliff J, Kansra S. Curcumin (diferuloylmethane) inhibits cell proliferation, induces apoptosis, and decreases hormone levels and secretion in pituitary tumor cells. Endocrinology 2008;149:4158-67.

263. Wang SL, Li Y, Wen Y, Chen YF, Na LX, Li ST, et al. Curcumin, a potential inhibitor of up-regulation of TNF-alpha and IL-6 induced by palmitate in 3T3-L1 adipocytes through NF-kappaB and JNK pathway. Biomed Environ Sci 2009;22:32-9.

264. Tominaga S, Sugahara T, Nishimoto S, Yamawaki M, Nakashima $\mathrm{Y}$, Kishida T, et al. The effect of Seco iso lariciresinol on 3T3-L1 adipocytes and the relationship between molecular structure and activity. Biosci Biotechnol Biochem 2009;73:35-9.

265. Kim MS, Kim JK, Kim HJ, Moon SR, Shin BC, Park KW, et al. Hibiscus extract inhibits the lipid droplet accumulation and adipogenic transcription factors expression of 3T3-L1 preadipocytes. J Alternative Complementary Med 2003;9:499-504.

266. Hwang JT, Park IJ, Shin JI, Lee SK, Baik HW, Ha J, et al. Genistein, EGCG, and capsaicin inhibit adipocyte differentiation process via activating AMP-activated protein kinase. Biochem Biophys Res Commun 2005;338:694-9. 
267. Okada T, Nakai M, Maeda H, Hosokawa M, Sashima T, Miyashita K. Suppressive effect of neoxanthin on the differentiation of 3T3-L1 adipose cells. J Oleo Sci 2008;57:345-51.

268. Yang JY, Della-Fera MA, Baile CA. Guggulsterone inhibits adipocyte differentiation and induces apoptosis in 3T3-L1 cells Obesity 2008;16:16-22.

269. Jiang L, Zhang NX, Mo W, Wan R, Ma CG, Li X, et al. Rehmannia inhibits adipocyte differentiation and adipogenesis. Biochem Biophys Res Commun 2008;371:185-90

270. Zong W, Zhao G. Corosolic acid isolation from the leaves of Eriobotrtajaponica showing the effects on carbohydrate metabolism and differentiation of 3T3-L1 adipocytes. Asia Pac J Clin Nutr 2007;16:346-52.

271. Oben JE, Ngondi JL, Blum K. Inhibition of Irvingia gabonensis seed extract (OB131) on adipogenesis as mediated via down regulation of the PPAR gamma and leptin genes and upregulation of the adiponectin gene. Lipids Health Dis 2008;7:1-6.

272. Langin D. Adipose tissue lipolysis as a metabolic pathway to define pharmacological strategies aginst obesity and the metabolic syndrome. Pharmacol Res 2006;53:482-91.

273. Rong X, Kim MS, Su N, Wen S, Matsuo Y, Yamahara J, et al. An aqueous extract of Salacia oblonga root, a herb-derived peroxisome proliferator-activated receptor alpha activator, by oral gavage over $28 \mathrm{~d}$ induces gender-dependent hepatic hypertrophy in rats. Food Chem Toxicol 2008;46:2165-72.

274. Huang TH, Peng G, Li GQ, Yamahara J, Roufogalis BD, Li Y. Salacia oblonga root improves postprandial hyperlipidemia and hepatic steatosis in zucker diabetic fatty rats: activation of PPAR-a. Toxicol Appl Pharmacol 2006;210:225-35.

275. Pang J, Choi Y, Park T. Ilex paraguariensis extract ameliorates obesity induced by high-fat diet: potential role of AMPK in the visceral adipose tissue. Arch Biochem Biophys 2008;476:178-85.

276. Lee J, Chae K, Ha J, Park BY, Lee HS, Jeong S, et al. Regulation of obesity and lipid disorders by herbal extracts from Morus alba, Melissa officinalis, and Artemisia capillaries in high-fat-dietinduced obese mice. J Ethnopharmacol 2008;115:263-70.

277. Lee JS, Lee MK, Ha TY, Bok SH, Park HM, Jeong KS, et al. Supplementation of whole persimmon leaf improves lipid profiles and suppresses body weight gain in rats fed high-fat diet. Food Chem Toxicol 2006;44:1875-83.

278. Weisberg SP, Leibel R, Tortoriello DV. Dietary curcumin significantly improves obesity-associated inflammation and diabetes in mouse models of diabesity. Endocrinology 2008;149:3549-58.

279. Manjunatha $H$, Srinivasan K. Hypolipidemic and antioxidant effects of curcumin and capsaicin in high-fat-fed rats. Can J Physiol Pharmacol 2007;85:588-96.

280. Asai A, Miyazawa T. Dietary curcuminoids prevent high-fatdiet-induced lipid accumulation in rat liver and epididymal adipose tissue. J Nutr 2001;131:2932-5.

281. Park SA, Choi MS, Kim MJ, Jung UJ, Kim HJ, Park KK, et al. Hypoglycemic and hypolipidemic action of Du-zhong
(Eucommia ulmoides Oliver) leaves water extract in C57BL/KsJ-db/db mice. J Ethnopharmacol 2006;107:412-7.

282. Kim SO, Yun SJ, Jung B, Lee EH, Hahm DH, Shim I, et al. Hypolipidemic effects of curde extract of adlay seed (Coix lachrymajobi var Mayuen) in obesity rat fed high-fat diet: relations of TNF- $\alpha$ and leptin mRNA expressions and serum lipid levels. Life Sci 2004;75:1391-404.

283. Yoshikawa M, Shimoda H, Nishida N, Takada M, Matsuda H. Salacia reticulata and its polyphenolic constituents with lipase inhibitory and lipolytic activities have mild anti-obesity effects in rats. J Nutr 2002;132:1819-24.

284. Nakagawa K, Kishida H, Arai N, Nishiyama T, Mae T. Licorice flavonoids suppress abdominal fat accumulation and increase in blood glucose level in obese diabetic KK-Ay mice. Biol Pharm Bull 2004;27:1775-8.

285. Mae T, Kishida H, Nishiyama T, Tsukagawa M, Konishi E, Kuroda M, et al. A licorice ethanolic extract with peroxisome proliferator-activated receptor-c ligand-binding activity affects diabetes in KK-Ay mice, abdominal obesity in diet-induced obese C57BL mice and hypertension in spontaneously hypertensive Rats. J Nutr 2003;133:3369-77.

286. Kobayashi Y, Nakano Y, Kizaki M, Hoshikuma K, Yokoo Y, Kamiya T. Capsaicin-like anti-obese activities of evodiamine from fruits of Evodia rutaecarpa, a vanilloid receptor agonist. Planta Med 2000;67:628-33.

287. Kim HJ, Bae IY, Ahn CW, Lee HG. Purification and identification of adipogenesis inhibitory peptide from black soybean protein hydrolysate. Peptides 2007;28:2098-103.

288. Yang JY, Lee SJ, Park HW, Cha YS. Effect of genistein with carnitine administration on lipid parameters and obesity in C57Bl/6J mice fed a high-fat diet. J Med Food 2006;9:459-67.

289. Morimoto C, Satoh Y, Hara M, Inoue S, Tsujita T, Okuda H. Antiobese action of raspberry ketone. Life Sci 2005;77:194-204.

290. Roffey BW, Atwal AS, Johns T, Kubow S. Water extracts from Momordica charantia increase glucose uptake and adiponectin secretion in 3T3-L1 adipose cells. J Ethnopharmacol 2007;112:77-84.

291. Hsu HK, Yang YC, Hwang JH, Hong SJ. Effects of Toona sinensis leaf extract on lipolysis in differentiated 3T3-L1 adipocytes. Kaohsiung J Med Sci 2003;19:385-9.

292. Sheng X, Zhang Y, Gong Z, Huang C, Zang YQ. Improved insulin resistance and lipid metabolism by cinnamon extract through activation of peroxisome proliferator-activated receptors. PPAR Res 2008:1-9. Doi:10.1155/2008/581348

\section{How to cite this article}

- Mani Kumar Bollapragada, Manjula Shantaram, Sunil Kumar R. Obesity: development, epidemiology, factors affecting, quantity, health hazards, management and natural treatment a review. Int J Pharm Pharm Sci 2017;9(2):12-26. 